\title{
EACD: evolutionary adaptation to concept drifts in data streams
}

\author{
Hossein Ghomeshi ${ }^{1}\left[\right.$ Mohamed Medhat Gaber $^{1} \cdot$ Yevgeniya Kovalchuk $^{1}$
}

Received: 10 December 2017 / Accepted: 14 January 2019 / Published online: 13 February 2019

(c) The Author(s) 2019

\begin{abstract}
This paper presents a novel ensemble learning method based on evolutionary algorithms to cope with different types of concept drifts in non-stationary data stream classification tasks. In ensemble learning, multiple learners forming an ensemble are trained to obtain a better predictive performance compared to that of a single learner, especially in non-stationary environments, where data evolve over time. The evolution of data streams can be viewed as a problem of changing environment, and evolutionary algorithms offer a natural solution to this problem. The method proposed in this paper uses random subspaces of features from a pool of features to create different classification types in the ensemble. Each such type consists of a limited number of classifiers (decision trees) that have been built at different times over the data stream. An evolutionary algorithm (replicator dynamics) is used to adapt to different concept drifts; it allows the types with a higher performance to increase and those with a lower performance to decrease in size. Genetic algorithm is then applied to build a two-layer architecture based on the proposed technique to dynamically optimise the combination of features in each type to achieve a better adaptation to new concepts. The proposed method, called EACD, offers both implicit and explicit mechanisms to deal with concept drifts. A set of experiments employing four artificial and five real-world data streams is conducted to compare its performance with that of the state-of-the-art algorithms using the immediate and delayed prequential evaluation methods. The results demonstrate favourable performance of the proposed EACD method in different environments.
\end{abstract}

Responsible editor: Jesse Davis, Elisa Fromont, Derek Greene, Björn Bringmann.

Hossein Ghomeshi

Hossein.Ghomeshi@mail.bcu.ac.uk

Mohamed Medhat Gaber

Mohamed.Gaber@bcu.ac.uk

Yevgeniya Kovalchuk

Yevgeniya.Kovalchuk@bcu.ac.uk

1 School of Computing and Digital Technology, Birmingham City University, Birmingham, UK 
Keywords Data streams · Ensemble learning · Concept drifts · Evolutionary algorithms · Genetic algorithm · Non-stationary environments

\section{Introduction}

A considerable effort of recent research has focused on data stream classification tasks in non-stationary environments (Gama et al. 2014). The main challenge in this research area concerns the adaptation to concept drifts, that is, when the data distribution changes over time in unforeseen ways. Concept drifts occur in different forms and can be divided into four general types: abrupt (sudden), gradual, incremental and recurrent (reoccurring). In abrupt (sudden) concept drifts, the data distribution at the time $t$ suddenly changes to a new distribution at the time $t+1$. Incremental concept drifts occur when the data distribution changes and stays in the new distribution after going through some new, unstable, median data distributions. In gradual concept drifts, the proportion of new probability distribution of incoming data increases, while the proportion of data that belong to the former probability distribution decreases over time. Recurring concept drifts happen when the same old probability distribution of data reappears after some time of a different distribution.

Ensemble learning has proved superiority for stream classification in non-stationary environments over other classification techniques (Gomes et al. 2017a; Krawczyk et al. 2017). Ensemble learning is a machine learning approach, in which predictions of individual classifiers are combined using a combination rule to predict incoming instances more accurately. The advantage of using ensemble learning techniques in non-stationary data stream classification lies in their ability to update swiftly according to the most recent data instances. This is usually achieved by training the existing classifiers in the ensemble and changing their weights according to their performance: adding new, better performing classifiers, and removing outdated, low performing classifiers. Applications of classification in non-stationary data streams include spam filtering systems, stock market prediction systems, fraud detection in banking networks, weather forecasting systems, data analysis in Internet of Things (IoT) networks, traffic and forest monitoring systems, among many others. The extensive range of applications makes the task of non-stationary data stream classification even more challenging, as various applications seek diverse purposes and have different conditions.

To propose a versatile yet robust ensemble approach in this context, the following main features should be taken into consideration:

- Accuracy the main target of any approach is usually to achieve a minimum misclassification rate. Hence, the average accuracy rate of an approach should be satisfactory in different evolving data streams.

- Efficiency in many applications, there are constraints on the system in terms of time and memory usage. When the time calculating an output or the amount of available memory is limited, the learning time and computational complexity of an approach should be minimised.

- Adaptation when a concept drift happens in a data stream, the accuracy of the ensemble decreases due to the change of the data distribution and the target concept. 
It is important to minimise the rate of misclassification and the time of recovery upon different types of concept drifts.

The majority of the existing ensemble methods are either focused on one or two of the aforementioned factors, or concentrate on a specific type of data streams. For instance, some approaches do not remove old classifiers (Elwell and Polikar 2011; Ramamurthy and Bhatnagar 2007); hence, the number of classifiers is unbounded, which can cause a low efficiency in terms of time and memory usage. Other approaches are designed to cope with recurring concept drifts only (Gonçalves Jr and De Barros 2013); therefore, such algorithms are only suitable for a limited number of applications and environments.

To overcome these limitations, we propose a novel ensemble learning method for data stream classification in non-stationary environments, called $E A C D$, that uses random selection of features and two evolutionary algorithms, namely, Replicator Dynamics (RD) and Genetic algorithm (GA). We train an ensemble of different classification types that consist of randomly drawn features (subspaces) of the target data stream. These randomly drawn subspaces are then optimised using GA to cope with different concept drifts over time. Training of the proposed ensemble is performed on sequential data blocks in the stream. The proposed ensemble technique allows a dynamic set of classification types to take action over time. In addition, the number of decision trees in a classification type (subspace) depends on the performance of this type on the most recent data. Hence, well performing types increase in size, while poorly performing types decrease in size.

In summary, our solution allows the ensemble to handle different types of concept drifts by employing two different evolutionary techniques. RD is used to continuously determine well and poorly performing types and expand or shrink them accordingly. GA is used to compose new, improved types out of the existing ones by iterating over the most recent data.

The rest of this paper is organised as follows. Section 2 presents an overview of related research. Section 3 describes our proposed method in detail and provides theoretical justification of the proposed method. Section 4 outlines the experimental set up and the results of comparing the proposed approach to other state-of-the-art methods. Section 5 comprehensively discusses the results of the experiments. Finally, conclusions and future work are presented in Sect. 6.

\section{Related work}

\subsection{Ensemble learning in non-stationary data streams}

The majority of the existing data stream learning approaches to non-stationary environments uses ensemble learning techniques for classification tasks (Chu and Zaniolo 2004a; Gama et al. 2014; Gomes et al. 2017a; Krawczyk et al. 2017), which are more flexible and trustworthy compared to single classifier techniques that use only one classifier for the task. 
The existing ensemble methods can be categorised into explicit and implicit methods. Explicit methods use a concept drift detection mechanism and have an explicit (immediate) reaction to a drift when it is detected, while implicit methods do not have an immediate reaction to concept drifts, and as such, adapt to drifts implicitly by updating the state of the ensemble according to the most recent instances.

\section{Implicit methods}

Online bagging (OzaBag) (Oza 2005) is an online version of bagging learning mechanism that can be used in data streams (as opposed to the standard bagging technique that requires the training set to be available at once). It updates each classifier in the ensemble with $k$ copies of the newly received instances. The value of $k$ comes from the Poisson distribution Poisson(1).

Online boosting (OzaBoost) (Oza 2005) is an online version of the boosting learning mechanism. In this method, every new example received by the ensemble is used to update all classifiers in a sequential manner. In other words, the first classifier is assigned with the highest possible weight for the newly received data, while the weights assigned to the next classifiers are based on the outcome of the previous classifiers.

OSBoost (Chen et al. 2012) is an algorithm that uses online boosting and combines weak learners by producing a connection between the online boosting and the batch boosting algorithms. It is theoretically proved to achieve a small error rate, as long as the number of weak learners and the number of examples are sufficiently large.

Dynamic Weighted Majority (DWM) (Kolter and Maloof 2007) is an implicit approach, where data come in an online form and get classified immediately. If a classifier misclassifies an instance after a predefined period ( $p$ instances), the weight of this classifier is reduced by a constant value regardless of the ensemble's output and all weights are normalised. Then, the classifiers with the weights lower than a predefined threshold $(\theta)$ are removed from the ensemble. Finally, when the whole ensemble misclassifies an instance, a new classifier is built and added to the ensemble. All classifiers are trained incrementally with incoming samples.

The Accuracy Updated Ensemble (AUE) algorithm (Brzezinski and Stefanowski 2014b) incrementally trains all old classifiers and weights them based on their error in a constant time and memory. In this algorithm, the incremental nature of Hoeffding trees (Domingos and Hulten 2000a) is combined with a normal block-based weighting mechanism. This approach does not remove any old classifiers; therefore, a threshold for memory is assigned so that whenever it is met, a pruning method is used to reduce the size of classifiers. An online version of this approach (OAUE) was introduced by the same authors (Brzezinski and Stefanowski 2014a).

Anticipative Dynamic Adaptation to Concept Changes (ADACC) (Jaber 2013) is an implicit method that attempts to optimise stability of the ensemble by recognising incoming concept changes. This is achieved by establishing an enhanced forgetting strategy for the ensemble. ADACC takes snapshots of the ensemble when a concept is recognised as stable and uses them when there is instability in the system to cope with concept drifts.

Social Adaptive Ensemble (SAE) (Gomes and Enembreck 2013) is a method that has the same learning strategy as the DWM algorithm. It maintains an ensemble 
that is arranged as a network (undirected graph) of classifiers. Two classifiers are connected to each other when they produce similar predictions. These connections are weighted according to a similarity coefficient equation. The ensemble is updated after a predefined number of instances. The same authors extended their method to SAE2 approach (Gomes and Enembreck 2014).

The main issue with implicit methods is that in most cases adaptation to a new concept takes a long time due to their implicit behaviour. Furthermore, concept drifts are not identified immediately with such approaches.

\section{Explicit methods}

Adaptive Boosting (Aboost) (Chu and Zaniolo 2004a) is one of the approaches that uses a concept drift detection method. It builds one classifier per every block of data that is received from the stream and classifies the instances. Then, it evaluates the ensemble's output and updates the weights of all classifiers based on whether or not an instance is classified correctly by the ensemble, as well as the classifier itself. Whenever a concept drift is detected, the weight of each classifier in the ensemble is reset to one. Finally, once the size of the ensemble is exceeded, the oldest classifier is removed from it.

Adwin Bagging (AdwinBag) (Bifet et al. 2009) is an approach that uses Oza's online bagging algorithm (Oza 2005) for its learning mechanism and adds a concept drift detector called ADaptive WINdowing (ADWIN) (Bifet and Gavalda 2007) to specify when a new classifier is required. AdwinBag is enhanced in the Leveraging Bagging (LevBag) algorithm (Bifet et al. 2010b) by the same authors. LevBag aims to add randomisation to the input and the output of the classifiers and increase the extent of re-sampling in the bagging technique. The re-sampling rate in LevBag is changed from Poisson(1) to Poisson ( $\lambda$ ), where $\lambda$ is a user defined parameter.

Yet another explicit approach is Recurring Concept Drift (RCD) (Gonçalves Jr and De Barros 2013). It uses a buffer to store the context of each data type in the stream. This framework contains a two-phase concept drift detection mechanism. First, a new classifier is created and trained alongside a new buffer when the drift detection mechanism signals a warning. If it then signals a drift, which means the concept drift is approved, the system checks whether or not the new concept is similar to another concept that has been previously stored in the buffer. If there has been a recurring concept drift, RCD uses the classifier created with that concept drift to classify the incoming data and then starts training the classifier. If no similar concept drift is found in the buffer, RCD stores the newly trained buffer and the classifier in the system and uses them to classify the incoming instances. If the system does not get the drift signal to approve the drift, it assumes it to be a false alarm; the system ignores the stored data and continues to classify using the current classifier. Note, only one classifier is activated at a time in this approach, while the rest are deactivated, unless the same data concept happens again.

Adaptive Random Forest (ARF) (Gomes et al. 2017b) is an explicit ensemble learning technique, which is an adaptation of the classical Random Forest algorithm (Breiman 2001) that grows decision trees by training them on re-sampled versions of the original data and by randomly selecting a small number of features that can 
be inspected at each node for split. ARF is based on a warning and drift detection scheme per tree, such that after a warning has been detected for one tree, another one (background tree) starts growing in parallel and replaces the original tree only if the warning escalates to a drift.

In summary, the main issue with explicit methods is their sensitivity to false alarms (noise). Therefore, accuracy of the system using such methods can be degraded severely by a wrongly detected concept drift. Furthermore, employing a good drift detection mechanism that can recognise different types of concept drifts (gradual, recurring, abrupt and incremental) (Gama et al. 2014) is a difficult task. In this scenario, RD offers a smooth yet effective way to improve the performance of the ensemble by increasing or reducing the number of trees in classification types. Furthermore, the main issue with implicit algorithms is their slowness in coping with concept drifts as they do not have an immediate reaction to drifts. This is the reason for using a concept drift detection algorithm along with GA to immediately react to concept drifts and to optimise the combination of the features in classification types. Overall, by combining $\mathrm{RD}$ with concept drift detection methods and GA, it is feasible to have the advantages of explicit and implicit methods alongside in the ensemble.

\subsection{Evolutionary algorithms in non-stationary data streams}

Evolutionary algorithms cannot be applied in their original state to the problems in streaming applications since the whole set of instances is not accessible to the stream processing system. However, such algorithms can be adapted to streaming data in different ways, e.g. the following algorithms are proposed in the literature for nonstationary data stream classification.

The StreamGP algorithm (Folino et al. 2007) builds an ensemble of classifiers using Genetic Programming along with the boosting algorithm to generate decision trees, each trained on different parts of the data stream. This algorithm is an explicit algorithm that uses a concept drift detection mechanism. Whenever a concept drift is detected, a new classifier is created using CGPC (Folino et al. 2006), which is a cellular genetic programming method that generates a classifier as a decision tree. Each population in this algorithm is a set of individual data blocks (nodes) that initially is drawn randomly. The newly created classifier is then added to the ensemble and all classifiers are boosted by updating their weights. This algorithm is different from our proposed algorithm in that in StreamGP, the aim of the optimisation technique is to find the best set of data blocks to create a new classifier. In $E A C D$ however, the aim of GA is to find the best combination of features to create new classification types. Unlike our method, the problem with StreamGP is that no new classifier is created by the system unless a concept drift is detected. This might negatively affect the performance upon incremental and gradual concept drifts that are hard to detect.

Online Genetic Algorithm (OGA) (Vivekanandan and Nedunchezhian 2011) is a rule-based learning algorithm that builds and updates a set of candidate rules for a data stream based on the evolution of the data stream itself. In this algorithm, the rules are initially set randomly, and after fully receiving a new data block, an iteration of GA is performed to search for new (better) candidate rules for all classes in the received data 
block. This process is repeated until the end of the stream. The differences between OGA and our proposed algorithm are as follows. Primarily, OGA is a rule-based learning algorithm, whereas $E A C D$ is an ensemble learning algorithm. The aim of GA in OGA is to create new rules or update the current rules, whereas the aim of GA in EACD is to optimise the classification types inside the ensemble. Furthermore, the iterations in OGA are performed over different data blocks (an iteration per each data block) and GA never stops its iterations (the maximum number of generations is unlimited), whereas in $E A C D$, the iterations are performed over the same fixed data in the buffer for each round of GA, and the number of generations is limited. The main issue with OGA is the long time it takes to adapt to new concept drifts since GA takes only one data block at each iteration, potentially requiring a large number of iterations to completely cope with a concept drift.

\section{Proposed method}

\subsection{Replicator dynamics: an overview}

RD is a simple model of evolution and prestige-biased learning in game theory (Bomze 1983; Hofbauer and Sigmund 2003). It provides a solution for selecting useful types from a population of diverse types. In this model, the act of selection happens at discrete times and 'the population of each type in the next selection is given by the replicator equation as a function of the type's payoff and its current proportion in the population' (Fawgreh et al. 2015). In other words, a type's expected payoff is determined by the payoff matrix, and hence, the population of each type is determined according to its expected payoff. The types that score above the average payoff increase in population, while the types that score below the average payoff decrease in population. The Replicator Equation is represented by the following formula:

$$
\dot{x_{i}}=x_{i}\left[(W x)_{i}-x^{T} W x\right]
$$

where $(W x)_{i}$ is the expected payoff for an individual and $x^{T} W x$ is the average payoff in the population state $x$.

In our proposed method, a type (classification type) is a subspace of the total number of features of the target data stream that initially is drawn randomly and then is being optimised using GA. A type's payoff is the average accuracy of the classifiers that have been built using the specified type (subspace of features). The expected payoff is the average accuracy of all classifiers in the ensemble.

\subsection{Genetic algorithm: an overview}

GA is a meta-heuristic algorithm inspired by the process of natural selection, which is a subset of a bigger class of algorithms called evolutionary algorithms. Such algorithms are commonly used to generate high-quality solutions to optimisation and search problems relying on bio-inspired operators such as mutation, crossover and selection. 
The reason for using GA in the proposed method is that GA is superior to other optimisation methods when there are a relatively large number of local optima (Elyan and Gaber 2017), which is the case in this problem, where numerous subspaces of features likely to form 'types' can form local optima.

The typical GA works as follows. A population is created from a group of individuals randomly. The individuals in the population are then evaluated. The evaluation function is provided by the programmer and gives the individuals a score based on how well they perform at the given task. Some individuals are then selected based on their fitness; the higher the fitness, the higher the chance of being selected. These individuals then reproduce to create one or more offspring, after which the offspring are mutated randomly. This continues until a suitable solution is found or the maximum number of generations is reached (Mantri et al. 2011). Figure 1 demonstrates how such a typical GA works. In our proposed method, the Initial Population is a random subspace of features (types) that have been drawn earlier, and the evaluation is performed by calculating the average accuracy of each subspace (type). Selection, Crossover and Mutation, as employed in our method, are discussed later in Sect. 3.3.

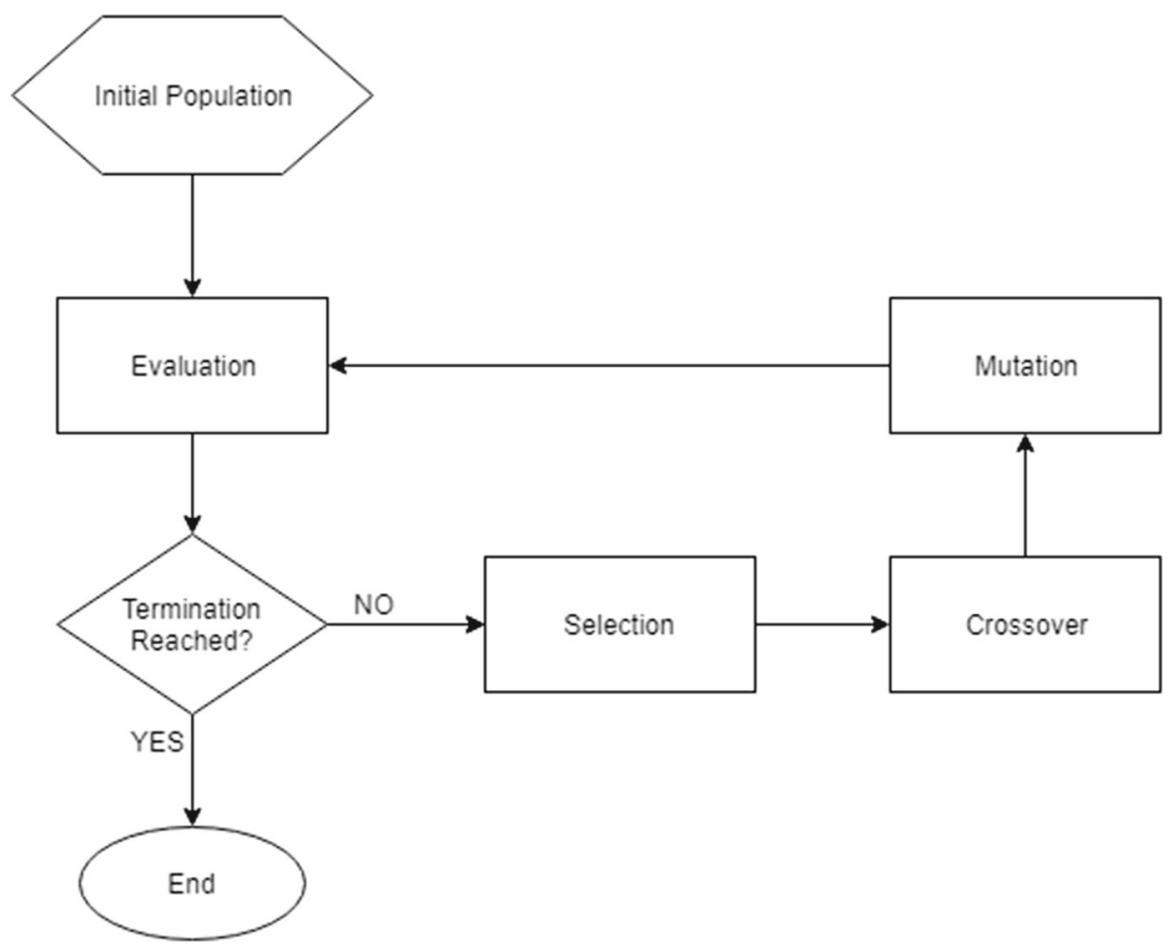

Fig. 1 An illustration of a typical genetic algorithm 


\subsection{EACD: evolutionary adaptation to concept drifts}

We propose a novel ensemble learning algorithm that is suitable for non-stationary data stream classification. In this algorithm, the data come as continuous data blocks. In this paper, each data block consists of 1000 samples that are selected arbitrarily; however, it can be set to any other values as required. The algorithm comprises of two different layers called the base layer and the genetic layer. Each layer has a set of classifiers that classify the incoming data independently. The base layer is always active, whereas the genetic layer is only active when GA has made its generations and the types are mature enough. The classifiers that comprise the second (genetic) layer have more weight than the classifiers comprising the base layer to achieve optimality of the types.

The base layer is built using random selection of features and gets extended using RD. The genetic layer is built by applying the GA optimisation technique to the set of features randomly selected from the base layer and introduces a new set of classification types that gets optimised by the recent instances stored in the buffer. Both the base and the genetic layers are detailed in the following subsections. In addition, Fig. 2 illustrates how the proposed algorithm works.

The rationale behind the proposed architecture is as follows. The main problem with the aforementioned explicit methods (the ones that use a concept drift detection mechanism) is their sensitivity to false alarms. In addition, detecting some types of concept drifts (especially gradual and incremental) is a hard task; hence, the employed detection mechanisms might not detect such drifts or detect them with a delay. In this scenario, RD offers a smooth yet effective way to improve the performance of the ensemble by increasing and reducing the number of trees in the classification types. Furthermore, the main problem with the implicit algorithms (the ones without a concept drift detection mechanism) is their slowness in coping with concept drifts as they do not have an immediate reaction to drifts. This is the reason for using a concept drift detection algorithm along with GA to immediately react to concept drifts and optimise the combination of the features in classification types. Overall, by combining $\mathrm{RD}$ with concept drift detection methods and GA, it is feasible to have the advantages of explicit and implicit methods alongside in the ensemble, as previously discussed in Sect. 2.

\subsubsection{Base layer}

As shown in Fig. 2, the base layer uses a random selection of features (subspaces) to create a variety of classification types in the ensemble, which ensures the ensemble diversity. RD is then applied to make the proposed method compatible with nonstationary environments and to seamlessly adapt to the most current types of data and concepts. In other words, RD is used to increase the number of well-performing classification trees and reduce the number of unhelpful ones.

The base layer is built using the following steps. First, $p$ percent of all features are randomly selected from the pool of data features (attributes) of the target data stream. This phase is called random subspace. In other words, the total number of features 


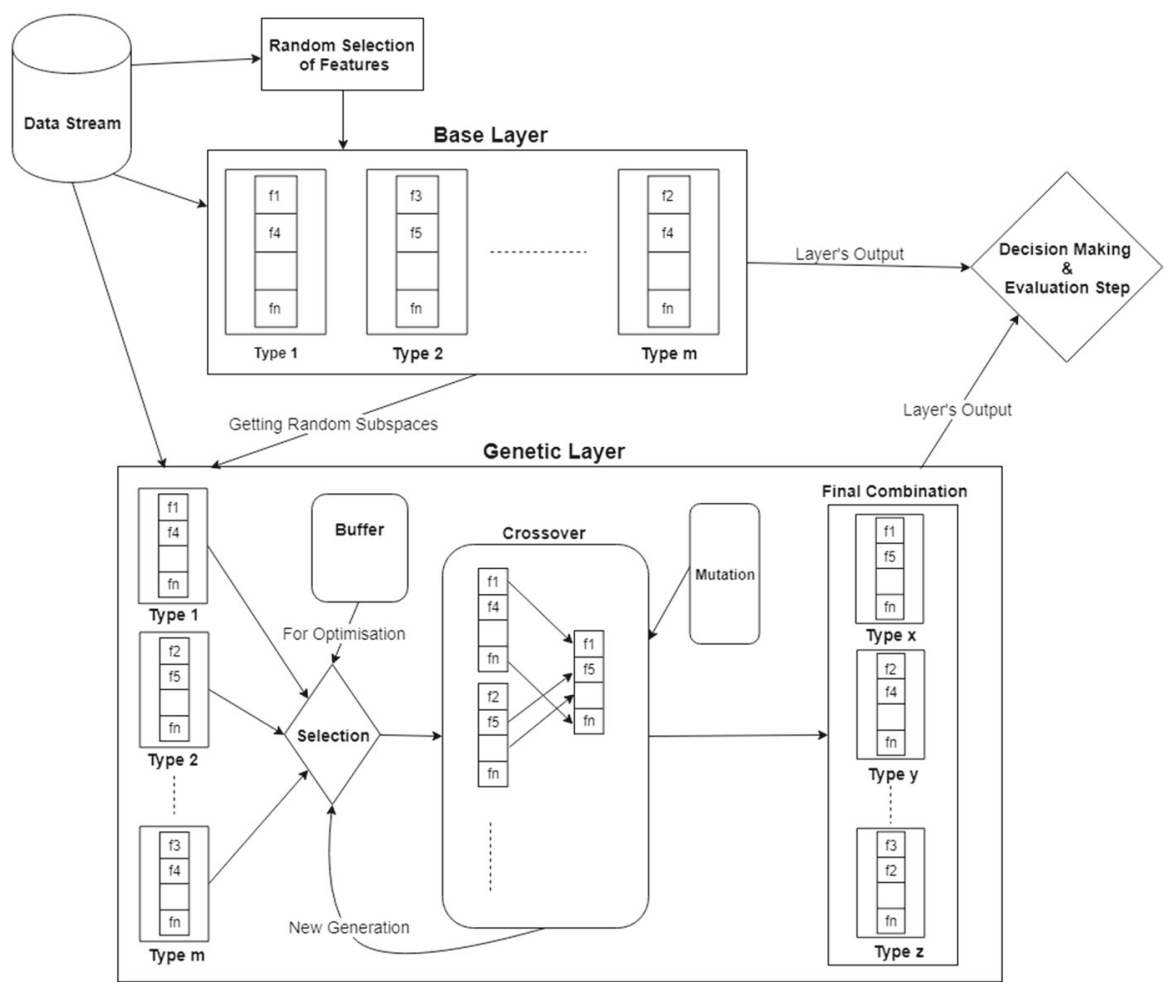

Fig. 2 Architecture of the proposed EACD algorithm

that is to be selected randomly from the pool of features is established as:

$$
n=\frac{p}{100} \times f
$$

where $n$ is the total number of features that needs to be selected, $p$ is an arbitrary number $(0<p<100)$ that shows the percentage of the features that should be selected randomly and $f$ is the total number of features of the target data stream. Each iteration of this step produces a set of randomly selected features (subspace) from the pool of features that we call a type. This step is repeated $m$ times; hence, there are $m$ independent classification types at the end of this step. Note that $m$ is a parameter of our proposed model for the total number of classification types in the ensemble and is chosen depending on the total number of features of the target stream; there should be a balance between the number of types $(m)$ and the number of features in each type $(p \times f)$.

Next, a decision tree is built per every classification type (subspace) when the first block of data (samples) is received by the system. Given the maximum number of classifiers for each type max, this step is repeated for the first $\frac{\max }{2}$ data blocks received by the system for the types to shape and reach a specific maturity level. This phase is called the initial training, during which, an average number of classifiers for every 
type in the ensemble is built. Note that for every data block received by the ensemble, all decision trees classify the instances and the majority voting then determines the ensemble's output. This is called the voting step.

Once the initial training phase is completed, each decision tree is evaluated after classifying incoming instances. The accuracy $(a)$ of each decision tree in a type is calculated as:

$$
a_{i}=\frac{c_{i}}{d b}
$$

where $c_{i}$ is the number of correctly classified instances in ith data block and $d b$ is the total number of instances in each data block. Accuracy of each type is the average accuracy of its related decision trees. Accuracy of the whole ensemble can be determined similar to Eq. 3. This phase is called the evaluation phase.

Next, the $R D$ stage is applied. This is when each type's accuracy (the average accuracy of all related trees) is taken into consideration and assessed with an expected payoff (explained previously in Sect. 3.1). The expected payoff in this paper is set to the average accuracy of all types in each data block. However, it can be determined in any other way, such as assigning a fixed number. The types with a higher payoff (accuracy) than the expected payoff get a new decision tree (i.e. a new decision tree is built for such data types based on the last block's samples), whereas the types with a lower payoff (accuracy) than the expected payoff lose a decision tree. In other words,

$$
\left\{\begin{array}{l}
a\left(t_{i}\right) \geq \frac{\sum_{i=1}^{m} a\left(t_{i}\right)}{m} \Rightarrow \text { grow } \\
a\left(t_{i}\right)<\frac{\sum_{i=1}^{m} a\left(t_{i}\right)}{m} \Rightarrow \text { shrink }
\end{array}\right.
$$

where $a\left(t_{i}\right)$ is the accuracy of the $i$ th type and $m$ is the total number of types.

Finally, every decision tree in the ensemble is trained with the samples from a newly received data block in the retraining phase. The purpose of this phase is to have a more updated ensemble, especially when a concept drift happens. In this situation, retraining can lead to a fast adaptation since all classifiers are trained with the newly evolved data.

To limit the size of the ensemble, an upper bound for the number of decision trees (classifiers) in a type is assigned. When the maximum size of a type is exceeded, the least performing decision tree of that specific type is removed. The upper bound (max) for the number of classifiers in this paper is set to the arbitrary value of $\max =20$. Furthermore, a lower bound $(\mathrm{min})$ is assigned to all types to prevent the types from complete removal. In this paper, the minimum size of all types is set to $\min =1$. Hence, a tree is not removed upon poor performance if it is the only one decision tree related to a type left.

Algorithm 1 shows how the base layer is built and works. In this algorithm, $t_{j}$ is the jshowshowthe type of the ensemble $(1 \leq j \leq m)$ and $a\left(t_{j}\right)$ is the accuracy of this type. The following functions are used in the presented algorithm:

- Classify(): the ensemble classifies data using the majority voting; 
- Evaluate(): evaluate the accuracy of all types in the ensemble using Eq. 3;

- Grow(): add a new classifier (decision tree) to the specified type (if Eq. 4 stands);

- Shrink(): remove one classifier (decision tree) from the specified type based on the ensemble's removal mechanism (if Eq. 4 stands); if this type has only one classifier, then do nothing;

- Train(): train all classifiers using the samples from the newly received data block.

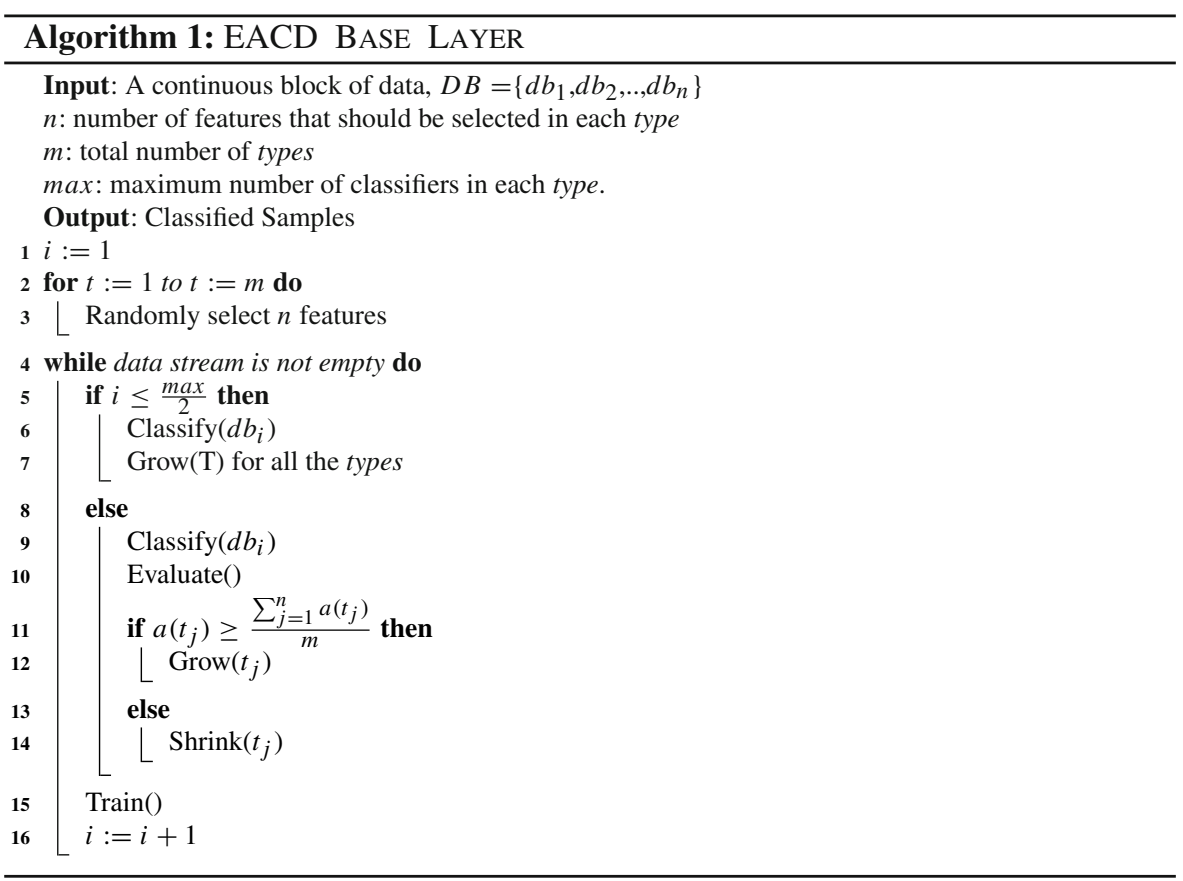

In the presented algorithm, lines 2 and 3 refer to the random subspace phase. Lines 5 , 6 and 7 are the initial training phase. The evaluation phase is implemented in line 10, the $R D$ phase is in lines 11 to 14, and finally, the retraining phase is in line 15. Decision trees are removed based on their performance; the tree that performs the worst in the specified type is removed.

\subsubsection{Genetic layer}

As demonstrated in Fig. 2, this layer is built using the existing classification types of the base layer. GA takes all randomly drawn classification types (subspaces) as its input and tries to form the best possible combination of the features in each type. This is achieved by iterating over a fixed data that has been received by the system recently (buffer). The genetic layer is different from the base layer only in this part (i.e. the combination of classification types), whereas the classification, training and updating mechanisms are the same as explained for the first layer. 
Algorithm 2 shows how the genetic layer is being built. First, the set of randomly drawn subspaces is taken from the base layer and considered as the first GA population. Note that in this algorithm, each classification type is considered as an individual in GA, and each feature inside a type is a chromosome of this individual.

The buffer always keeps the most recently labelled instances received by the system. It serves as a search space for the GA optimisation task. Whenever GA starts or restarts, it copies the data inside the buffer into the memory and uses them for its procedures, i.e. the selection stage and the fitness function.

Selection stage for every GA iteration, the classification types that have a better accuracy than the overall average accuracy of all types over the search space are selected for the crossover stage. Hence, the GA fitness function is the types' average accuracy over the search space. Algorithm 2 refers to this part with "Selection()" function.

Crossover stage the types selected in the selection stage are chosen for GA breeding purposes. This lets the types with a better accuracy to pair with other well performing types to make offspring. Algorithm 2 refers to this part with "Crossover()" function.

Mutation stage: the mutation rate of 5\% applies upon breeding of the types. Hence, there is a $5 \%$ chance for an offspring to get a random feature from the pool of features instead of getting all of them from its parents. Algorithm 2 refers to this part with "Mutation()" function.

When the maximum number of generations is achieved, the resulting classification types form a new set of classifiers that starts to be trained and evaluated with incoming data. The new ensemble model is said to be mature enough when its performance on the latest data block is better than the average performance of the algorithm. As mentioned before, the base layer is always active, whereas the genetic layer is active when the GA has done its job and the layer has reached its maturity level. All classifiers inside the base layer of the proposed algorithm are given the arbitrary weight of one $\left(W_{b}=1\right)$, whereas all classifiers inside the genetic layer are given the arbitrary weight of two $\left(W_{g}=2\right)$. This intensifies the effect of the genetic layer on the algorithm given the optimality of the types.

Once a new data block is received by the system, it goes to both layers, and the classifiers inside each layer classify the instances and send their predictions to the decision making part of the algorithm independently (as illustrated in Fig. 2). The decision maker then considers all the received predictions from the active classifiers and performs the voting procedure according to the weight of each prediction. This decision maker also tracks and keeps the average accuracy of each layer in the algorithm. Whenever GA is due to restart its procedures, the genetic layer is deactivated and cleared to make room for the new set of types. To determine when to start a new set of GA generations (i.e. reset the genetic layer), one implicit and one explicit mechanisms are proposed in this paper.

In the implicit mechanism, GA starts resetting the genetic layer when the base layer has proved to have the better average accuracy over the last arbitrarily set number of data blocks (we used 10 data blocks). This evaluation part is calculated continuously by the decision maker part mentioned previously in this section. In the implicit variants, the buffer inside the genetic layer stores the last data block received by the system. In the explicit mechanism, a concept drift detection method is utilised to specify when 
to reset the genetic layer. When the concept drift detector signals a drift, GA starts to rebuild its layer. In this paper, we used the early drift detection method (EDDM) (Baena-Garcia et al. 2006) as the explicit mechanism; however, any concept drift detection method can be used as the drift detector. EDDM is especially designed to improve the detection in presence of gradual concept drifts compared to other drift detection methods. The basic idea of EDDM is to consider the distance between two errors instead of considering only the number of errors in the classification process. In the explicit variants of the proposed method the buffer inside the genetic layer starts storing the instances once the concept drift detector signals a warning. Hence, when the drift detector signals a drift, the instances inside the buffer represents the new concept. Algorithm 2 refers to the Concept Drift Detection part with "DriftDetector()" function.

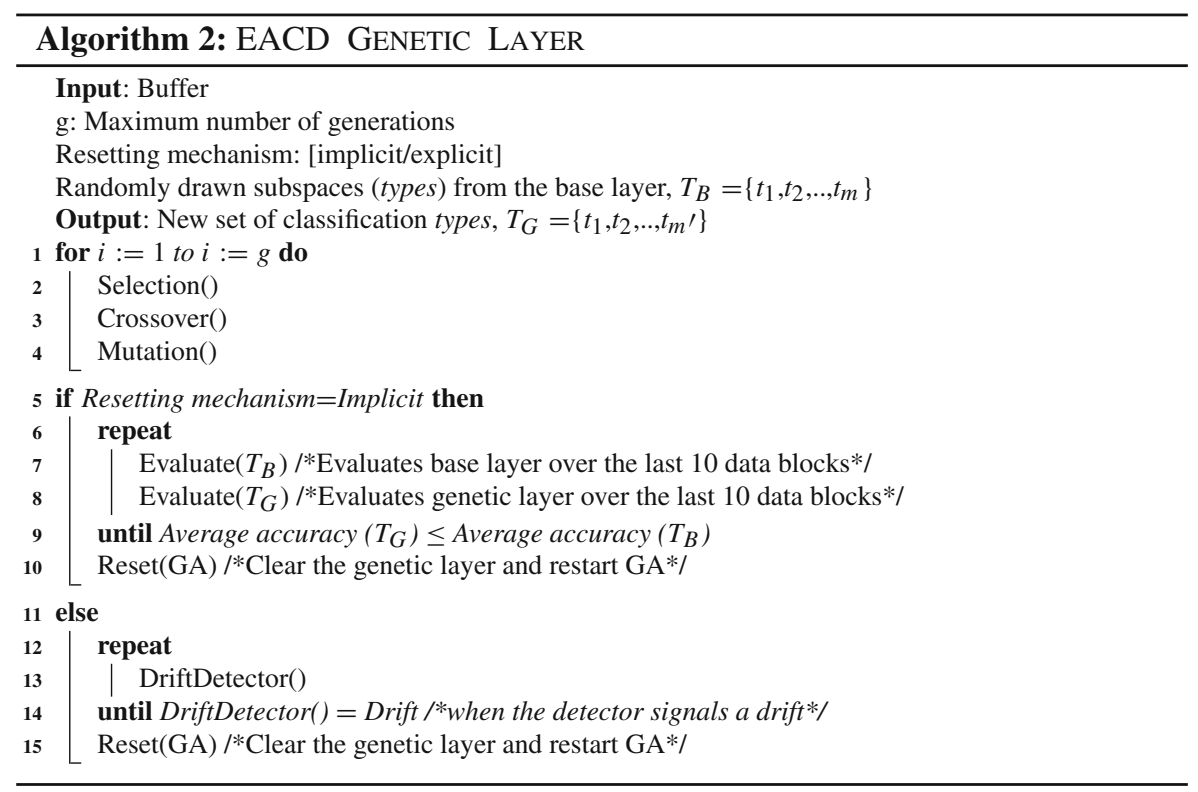

\subsection{Theoretical justification}

In the literature of mining non-stationary data streams, there is no deterministic method that can guarantee to find the global optima. This is due to the evolving nature of the data that come in the form of a stream. Hence, a single classifier of a data stream that is optimal in a specific environment can become the worst classifier once the data has evolved in the same data stream. By adding randomisation to create different classification types in the first layer of the proposed method, it is feasible to have a variety of classifiers in the ensemble. This leads to a diverse set of available solutions to quickly cope with an occurring concept drift. However, having different classification 
types can also cause problems such as degrading the accuracy in case of using one or more poor types. This problem is tackled by employing RD to increase the number of well-performing types and reduce the number of low-performing ones in the base layer of the proposed algorithm.

Furthermore, "stochastic search and optimisation pertains to problems where there is random noise in the measurements provided and/or there is injected randomness in the algorithms itself" (Spall 2005). Hence, GA is used in the second (genetic) layer to create new classification types to optimise the combination of features of the random types used in the first (base) layer. GA is a powerful and broadly applicable stochastic optimisation technique (Gen and Cheng 2000) that can be used in dynamic environments (e.g. data streams) after adding a few changes to its mechanism, as was proposed in this paper.

\section{Experimental study}

To evaluate the proposed algorithm, a set of experiments is conducted using nine datasets comprising of four artificial (synthetic) data stream generators and five realworld data streams. We compare the EACD algorithm to the state-of-the-art ensemble methods for non-stationary data stream classification that have shown a good performance and reliable results (Brzezinski and Stefanowski 2014a; Gomes et al. 2017b), including Dynamic Weighted Majority (DWM) (Kolter and Maloof 2007), Online Accuracy Updated Ensemble (OAUE) (Brzezinski and Stefanowski 2014a), OSBoost (Chen et al. 2012), Leveraging Bag (LevBag) (Bifet et al. 2010b) and Adaptive Random Forest (ARF) (Gomes et al. 2017b).

EACD is developed in Java programming language using the Massive Online Analysis (MOA) API (Bifet et al. 2010a). All other algorithms are already included in the MOA framework (Bifet et al. 2010a), which is used as the experimental environment here. MOA is an open source framework for data stream mining in evolving environments. When running LevBag, ARF, DWM, OAUE and OSBoost, their default parameters as set in MOA are used, while the parameters for running the proposed algorithm are listed in Sect. 4.2.

To have a thorough set of experiments with precise results, 10 different variants for every artificial data stream are generated and each method is tested on all variants. These variants are generated by changing different parameters in all artificial streams. The selected parameters for each data stream generator are specified later in Sect. 4.1. For every real-world data stream, each experiment is repeated 10 times over the same data stream.

There are two different evaluation runs for each experiment. The first run involves passing one of the chosen datasets through a specific algorithm using the prequential evaluation technique with an immediate access to the real labels of the instances that have been assigned by the system. This evaluation run is called the immediate setting. The second run also involves passing each dataset through a specific algorithm using the prequential evaluation; however, the real labels of the instances are accessed with a delay. This evaluation technique, called the delayed setting, can provide more realistic experiments, since the actual labels of streaming data are usually not available 
immediately in the real world. The classification performance estimates are calculated in the same way for both the immediate and the delayed settings. For the delayed setting, the parameter of delay is set to an arbitrary value of 1,000; hence, the label of each instance is revealed after passing 1,000 instances. The window size (width) of the experiments is set to 1,000 for both the immediate and the delayed settings.

Hoeffding trees are used in the experiments as the base classifiers (decision trees). Hoeffding tree, also known as the Very Fast Decision Tree (VFDT) method (Domingos and Hulten 2000a), is an incremental decision tree algorithm that is capable of learning from massive data streams.

The experiments were performed on a machine equipped with an Intel Core i74702MQ CPU @ 2.20GHz and 8.00 GB of installed memory (RAM).

\subsection{Datasets}

\subsubsection{Artificial data streams}

The following four artificial data stream generators are employed to simulate data for the experiments: the SEA generator, the Hyperplane generator, the Random Tree (RT) generator and the LED generator. Ten different stream variants are created for each of the considered data generators using their respective parameters to examine the performance of the tested algorithms depending on the type of the concept drifts. In case of the SEA generator, the variants are built by changing the random seed along with the type of manually added concept drifts. For the Hyperplane generator, different variants are built by tweaking the number of drifting attributes and the magnitude of changes in data. For the RT generator, the random seed number along with the number of attributes and classes are changed. Finally, for the LED generator, different variants are built by tweaking the number of drifting attributes and the random seed number.

\section{SEA generator}

The SEA generator (Street and Kim 2001) is a synthetic data stream generator that aims to simulate concept drifts over time. It generates random points in a three-dimensional feature space; however, only the first two features are relevant.

In case of the SEA generator, each variant includes one million instances. In addition, different concept drifts are manually chosen to happen in the instance numbers $200 \mathrm{~K}, 400 \mathrm{~K}, 600 \mathrm{~K}$ and $800 \mathrm{~K}$. For the first five variants, two abrupt concept drifts with a width (width of concept drift change) of one are added at the instance numbers $200 \mathrm{~K}$ and $400 \mathrm{~K}$, and two recurrent concept drifts with the same width are added at the instance numbers $600 \mathrm{~K}$ and $800 \mathrm{~K}$. For the remaining five variants, two gradual concept drifts with a width of 10,000 are added at the instance numbers $200 \mathrm{~K}$ and $400 \mathrm{~K}$, and two recurrent concept drifts with the same width are added at the instance numbers $600 \mathrm{~K}$ and $800 \mathrm{~K}$. 
Table 1 The number of drifting attributes and the magnitude of change selected for different stream variants based on the Hyperplane generator

\begin{tabular}{lllcll}
\hline Variant & No. of drifting att. & Mag. of change & Variant & No. of drifting att. & Mag. of change \\
\hline 1 & 2 & 0.01 & 2 & 2 & 0.02 \\
3 & 3 & 0.01 & 4 & 3 & 0.02 \\
5 & 4 & 0.01 & 6 & 4 & 0.02 \\
7 & 5 & 0.01 & 8 & 5 & 0.02 \\
9 & 6 & 0.01 & 10 & 6 & 0.02 \\
\hline
\end{tabular}

Table 2 Total number of attributes, number of classes and random seed number of different stream variants based on the RT generator

\begin{tabular}{llllllll}
\hline Variant & Attributes & Classes & Seed no. & Variant & Attributes & Classes & Seed no. \\
\hline 1 & 10 & 2 & 1 & 2 & 10 & 2 & 2 \\
3 & 12 & 3 & 1 & 4 & 12 & 3 & 2 \\
5 & 14 & 4 & 1 & 6 & 14 & 4 & 2 \\
7 & 16 & 5 & 1 & 8 & 16 & 5 & 2 \\
9 & 18 & 6 & 1 & 10 & 18 & 6 & 2 \\
\hline
\end{tabular}

\section{Hyperplane generator}

The Hyperplane generator (Hulten et al. 2001) is an artificial data stream with drifting concepts based on hyperplane rotation. It simulates concept drifts by changing the location of the hyperplane. The smoothness of drifting data can be changed by adjusting the magnitude of the changes.

In the presented experiments, the number of classes and attributes are set to two and ten, respectively, and the number of drifting attributes and the magnitude of changes are set as indicated in Table 1. The number of instances in each stream is set to one million.

\section{Random tree generator}

The RT generator (Domingos and Hulten 2000a) builds a decision tree by randomly selecting attributes as split nodes and assigning random classes to them. After the tree is built, new instances are obtained through the assignment of uniformly distributed random values to each attribute. The leaf reached after a traverse of the tree determines its class value according to the attribute values of an instance. The RT generator allows customising the number of nominal and numeric attributes, as well as the number of classes. In the experiments, the number of classes, the number of features and the random seed number are chosen as indicated in Table 2. 


\section{LED generator}

LED (Breiman et al. 1984) is a well-known data stream generator. The goal here is to predict the next digit to be displayed on the LED display. The generator contains 24 Boolean features, 17 of which are irrelevant and the remaining seven features correspond to each segment of a seven-segment LED display. Each feature has a $10 \%$ chance of being inverted. In this paper, the LED generator is used to simulate concept drifts by swapping four of its features resulting in ten different stream variants. For the first five variants, the number of drifting attributes are chosen to be 1, 2, 3, 4 and 5 , respectively. For the next five variants, only the random seed is changed, while the drifting attributes remain the same as in the first five variants.

\subsubsection{Real world data streams}

\section{Forest cover-type dataset}

The Forest Cover-type data stream (Blackard and Dean 1999) is a real world dataset from the UCI Machine Learning Repository. ${ }^{1}$ It contains the forest cover type of $30 \times 30$ meter cells obtained from the US Forest Service (USFS). It consists of 581,012 instances and 54 attributes. The goal in this dataset is to predict the forest cover type from cartographic variables.

\section{Electricity dataset}

Electricity is a widely used dataset by Harries and Wales (1999) collected from the Australian New South Wales electricity market. In this market, prices are not fixed and affected by demand and supply. The Electricity dataset contains 45,312 instances. Each instance contains eight attributes, and the target class specifies the change of the price (whether it goes up or down) according to its moving average over the last 24 hours.

\section{Airlines dataset}

Airlines $^{2}$ is a regression dataset. The task is to predict whether a flight will be delayed providing the information on its scheduled departure. This dataset has two classes (whether a flight is delayed or not) and contains 539,383 records with seven attributes (three numeric and four nominal).

\section{Poker-hand dataset}

The Poker-Hand dataset from the UCI Machine Learning Repository ${ }^{3}$ consists of 1,000,000 instances and 11 attributes. Each record of the Poker-Hand dataset is an

\footnotetext{
$1 \mathrm{http} / / /$ archive.ics.uci.edu/ml.

2 http://kt.ijs.si/elena_ikonomovska/data.html.

3 https://archive.ics.uci.edu/ml/datasets/Poker+Hand.
} 
example of a hand consisting of five playing cards drawn from a standard deck of 52 . Each card is described using two attributes (suit and rank), with a total of 10 predictive attributes. There is one class attribute that describes the "poker hand".

\section{KDDcup99}

KDDcup99 (Cup 1999) is the dataset used in the "Third International Knowledge Discovery and Data Mining Tools Competition". The competition task was to build a network intrusion detector-a predictive model capable of distinguishing between "bad" connections (intrusions or attacks) and "good" (normal) connections. KDDcup99 contains a standard set of data to be audited, which includes a wide variety of intrusions simulated in a military network environment. This dataset contains 41 attributes and 23 classes.

\subsection{EACD variations}

Eight different variations of the proposed algorithm are implemented and compared in the experiments to evaluate the impact of each EACD characteristic and discuss the effect of employing different parameters in the EACD algorithm. The base variations only use the base layer of the proposed algorithm, while GA optimisation is not applied; only base 4 variation uses the concept drift detector to restart the layer upon drifts. The implicit ( $I m p$ ) variations use an implicit mechanism, whereas the explicit $(E x p)$ variations use an explicit mechanism to specify when the genetic layer should be restarted (as explained in Sect. 3.3.2). The specific parameters of the eight proposed variations are as follows:

- EACD base $: p=60 \%$ and $m=0.6 \times f$;

- EACD Dase $2: p=30 \%$ and $m=0.3 \times f$;

- EAC D base3: $p=60 \%$ and $m=0.3 \times f$;

- EAC $D_{\text {base } 4}: p=60 \%, m=0.6 \times f$ and restarting the ensemble upon drifts;

- EACD $D_{\text {Imp }}: g=15, z=5 \%, p=60 \%$ and $m=0.6 \times f$;

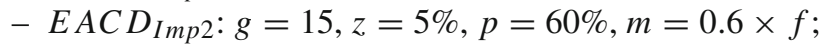

- EACD $D_{\text {Exp }}: g=15, z=5 \%, p=60 \%, m=0.6 \times f$;

- EAC $D_{E x p 2}: g=15, z=0 \%, p=60 \%, m=0.6 \times f$,

where $p$ is the number of features in each classification type, $m$ is the number of classification types in the layer, $f$ is the total number of features in the data stream, $g$ is the total number of generations for each GA iteration and $z$ is the mutation rate of GA.

\subsubsection{Computational complexity}

Assuming the number of classes $c$, the number of attributes in each classification type $p$, the values per attribute $v$ and the maximum number of trees in the ensemble $k$, no more than $p$ attributes are considered in a single Hoeffding tree (Domingos and Hulten 2000a). Each attribute at a node requires computing $v$ values. Since calculating 
information gain requires $c$ arithmetic operations, the cost of $k$ Hoeffding trees at each time-step in the worst case scenario is $\mathrm{O}(k c p v)$. Given the number of classification types in the ensemble $m$ and the fact that RD uses $m$ arithmetic operations to calculate payoffs, the cost of applying RD to the ensemble is only $\mathrm{O}(m)$. Hence, the time complexity of deploying the base variations of the proposed method ( $E A C D_{\text {base }}$, $E A C D_{\text {base } 2}$ and $\left.E A C D_{\text {base } 3}\right)$ is $\mathrm{O}(m+(k c v p))$.

Assuming the size $s$ of the GA population and the total number of generations $g$, the cost of GA optimisation is $\mathrm{O}(\mathrm{sg})$ at each time when the genetic layer needs to be restarted. Hence, the time complexity of deploying the implicit variations of the proposed method (EAC $D_{\text {Imp }}$ and $E A C D_{\text {Imp } 2)}$ is $\mathrm{O}(m+(k c v p)+(s g))$.

Finally, given $d$ as the number of instances in each data block and $f$ as the total number of features in the dataset, the EDDM drift detection method, which uses J48 (C4.5) decision tree as its learning mechanism, requires $\mathrm{O}\left(d f^{2}\right)$ of time. Hence, the time complexity of deploying the explicit variations of the proposed method (EACD Exp and $\left.E A C D_{\text {exp } 2}\right)$ is $\mathrm{O}\left(m+(k c v p)+(s g)+\left(d f^{2}\right)\right)$. Note that the cost of running evolutionary methods is minimised providing the variations applied to EACD as previously discussed.

\subsection{Results}

The considered algorithms are compared using standard criteria, including the classification accuracy and the overall time. There are two settings for each experiment (immediate and delayed) as explained previously in this section.

Tables 3 and 4 show the average accuracy for the proposed EACD variations over the mentioned nine datasets in the immediate and the delayed settings, respectively. As can be seen from the tables, E AC $D_{E x p}$ has the best average accuracy over the Hyperplane, the LED, the SEA, the Airlines, the Electricity and the Poker-Hand datasets. It also has the best overall average accuracy in both the immediate and the delayed settings. $E A C D_{\text {Imp }}$ has the best average accuracy over the Forest Cover-type and the RT datasets, wheras $E A C D_{E x p 2}$ has the best average accuracy over the KDDcup99 dataset.

As the difference between $E A C D_{\text {Imp }}$ and $E A C D_{\operatorname{Imp} 2}$ is in their number of generations used in each GA iteration, their accuracy is not significantly different, and $E A C D_{\text {Imp }}$, which has a higher number of generations (15), performs better over all datasets. It is clear that the evaluation time of $E A C D_{I m p 2}$ is less than that of $E A C D_{\text {Imp }}$ since GA performs faster on 10 generations compared to 15 generations. Similarly, as the difference between $E A C D_{E x p}$ and $E A C D_{E x p 2}$ is in their GA mutation rate parameter, they both have comparable accuracy and execution time, and only $E A C D_{E x p}$ accuracy is slightly better for the majority of the datasets.

Table 5 shows the overall evaluation time of the proposed EACD variations in

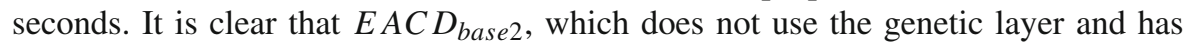
the lowest values of both $p$ and $m$ parameters, is the fastest variation. $E A C D_{\text {Imp }}$ and $E A C D_{\operatorname{Imp} 2}$ are slightly less time-consuming compared to $E A C D_{\operatorname{Exp}}$ and $E A C D_{E x p 2}$ because they do not use a concept drift detection algorithm. Finally, the evaluation times of $E A C D_{E x p}$ and $E A C D_{E x p 2}$ variations are similar as their only 
Table 3 Average accuracy $(\%)$ of the EACD variations in the immediate setting

\begin{tabular}{lllllllll}
\hline Dataset & base & base2 & base3 & base4 & Imp & Imp2 & Exp & Exp2 \\
\hline Hyper. & 86.31 & 77.47 & 80.52 & 84.74 & 89.23 & 88.54 & $\mathbf{9 0 . 5 9}$ & 90.53 \\
LED & 68.78 & 63.05 & 64.12 & 67.75 & 74.78 & 74.02 & $\mathbf{7 5 . 4 5}$ & 75.42 \\
RT & 88.03 & 79.34 & 86.34 & 89.93 & $\mathbf{9 1 . 8 9}$ & 91.23 & 91.42 & 91.41 \\
SEA & 87.35 & 82.43 & 84.56 & 88.90 & 87.43 & 85.78 & $\mathbf{9 0 . 0 8}$ & 90.00 \\
Airlines & 62.97 & 60.09 & 61.78 & 62.08 & 64.37 & 63.98 & $\mathbf{6 6 . 6 1}$ & 66.60 \\
Elec. & 81.01 & 77.34 & 80.45 & 81.76 & 90.30 & 90.23 & $\mathbf{9 2 . 1 4}$ & 92.10 \\
Forest & 83.56 & 70.34 & 80.67 & 85.83 & $\mathbf{9 2 . 6 4}$ & 91.94 & 91.73 & 91.73 \\
KDDcup & 99.76 & 98.67 & 98.89 & 99.76 & 99.76 & 99.76 & 99.78 & $\mathbf{9 9 . 7 9}$ \\
Poker & 80.24 & 73.45 & 75.23 & 79.51 & 83.45 & 82.78 & $\mathbf{8 6 . 2 1}$ & 86.17 \\
Overall average & 82.00 & 75.80 & 79.17 & 82.25 & 85.98 & 85.36 & $\mathbf{8 7 . 1 1}$ & 87.08 \\
\hline Best resit & & & & & & & &
\end{tabular}

Best results are given in bold

Table 4 Average accuracy $(\%)$ of the EACD variations in the delayed setting

\begin{tabular}{lcccccccc}
\hline Dataset & base & base2 & base3 & base4 & Imp & Imp2 & Exp & Exp2 \\
\hline Hyper. & 84.35 & 75.34 & 78.23 & 83.40 & 88.43 & 88.05 & $\mathbf{9 0 . 0 2}$ & 89.98 \\
LED & 68.17 & 62.67 & 64.00 & 67.69 & 73.60 & 73.14 & $\mathbf{7 5 . 2 6}$ & 75.25 \\
RT & 87.16 & 79.02 & 84.23 & 87.92 & $\mathbf{9 1 . 2 4}$ & 90.20 & 91.05 & 91.01 \\
SEA & 85.94 & 80.56 & 82.43 & 87.38 & 87.06 & 85.24 & $\mathbf{8 9 . 2 2}$ & 89.20 \\
Airlines & 60.45 & 56.07 & 58.12 & 62.48 & 62.18 & 62.56 & $\mathbf{6 3 . 3 5}$ & 63.14 \\
Elec. & 74.35 & 73.67 & 84.35 & 75.01 & 83.32 & 84.35 & $\mathbf{8 5 . 0 3}$ & 84.97 \\
Forest & 79.45 & 70.34 & 79.23 & 80.05 & $\mathbf{8 5 . 9 0}$ & 85.34 & 84.83 & 84.80 \\
KDDcup & 99.76 & 98.67 & 98.84 & 99.75 & 99.75 & 99.76 & 99.76 & $\mathbf{9 9 . 7 7}$ \\
Poker & 77.92 & 70.78 & 73.37 & 76.90 & 78.03 & 77.45 & $\mathbf{8 0 . 2 1}$ & 79.24 \\
Overall average & 79.73 & 74.12 & 78.09 & 80.06 & 83.28 & 82.90 & $\mathbf{8 4 . 3 0}$ & 84.15 \\
\hline Best results & & & & & & &
\end{tabular}

Best results are given in bold

difference is in the GA mutation rate, which does not affect the times severely. Note that the evaluation times do not have significant difference in the immediate and the delayed settings; hence, only the evaluation times of the immediate setting are shown in this paper.

Tables 6 and 7 show the average, minimum and maximum accuracy along with the standard deviation of the proposed EAC $D_{\text {Exp }}$ method compared to the other stateof-the-art methods over the nine datasets in the immediate and the delayed settings, respectively. The best results for each datset are highlighted in bold. In the immediate setting (Table 6), EAC $D_{\text {Exp }}$ has the best average accuracy over four datasets, LevBag performs the best over two datasets, while OAUE, OSBoost and ARF achieve the best accuracy over one dataset. In the delayed setting (Table 7), EAC $D_{\operatorname{Exp}}$ has the best average accuracy over five datasets, OAUE achieves the best performance over two datasets, while OSBoost and LevBag achieve the best accuracy over one dataset. 
Table 5 Average evaluation time (in seconds) of executing the EACD variations

\begin{tabular}{lllllllll}
\hline Dataset & base & base 2 & base3 & base4 & Imp & Imp2 & Exp & Exp2 \\
\hline Hyper. & 189 & $\mathbf{1 4 7}$ & 162 & 195 & 297 & 290 & 349 & 349 \\
LED & 183 & $\mathbf{1 4 3}$ & 149 & 186 & 419 & 417 & 423 & 420 \\
RT & 233 & $\mathbf{2 0 2}$ & 227 & 251 & 515 & 509 & 607 & 606 \\
SEA & 304 & $\mathbf{2 8 9}$ & 293 & 316 & 667 & 663 & 880 & 870 \\
Airlines & 228 & $\mathbf{2 1 6}$ & 220 & 232 & 665 & 659 & 657 & 651 \\
Elec & 5.80 & $\mathbf{4 . 6 7}$ & 5.08 & 6.00 & 9.54 & 9.54 & 10.45 & 10.40 \\
Forest & 756 & $\mathbf{5 4 7}$ & 694 & 756 & 887 & 860 & 935 & 917 \\
KDDcup & 305 & $\mathbf{2 9 1}$ & 303 & 319 & 435 & 430 & 536 & 536 \\
Poker & 240 & $\mathbf{2 0 5}$ & 225 & 273 & 319 & 317 & 346 & 346 \\
\hline
\end{tabular}

Best results are given in bold

Table 8 shows the overall evaluation CPU-time of the proposed $E A C D_{E x p}$ method compared to the other methods. For the majority of the datasets, DWM and OSBoost achieve the shortest evaluation time by far, while $E A C D_{E x p}$ has the longest evaluation time for the majority of the datasets.

Figure 3 demonstrates the behaviour of the proposed $E A C D_{E x p}$ method along with the other methods over the SEA data stream upon different concept drifts (abrupt, gradual and recurrent) that have been added manually to different stages of the data stream (instance numbers $200 \mathrm{~K}, 400 \mathrm{~K}, 600 \mathrm{~K}$ and $800 \mathrm{~K}$ ) in both the immediate (Fig. 3a-c, g) and the delayed (Fig. 3b, d, f, h) settings. In Fig. 3a, b, an abrupt concept drift centred in the instance number $200 \mathrm{~K}$ is added with a width of 1 . In Fig. 3c, d, a recurrent concept drift centred in the instance number $600 \mathrm{~K}$ is added with a width of 1 . In Fig. 3e, $\mathrm{f}$, a gradual concept drift centred in the instance number $400 \mathrm{~K}$ is added with a width of 10,000. And finally in Fig. 3g, h, a recurrent concept drift centred in the instance number $800 \mathrm{~K}$ is added with a width of 10,000 .

\subsection{Statistical analysis}

The Friedman test (Friedman 1940) is a non-parametric statistical test similar to the parametric repeated measures ANOVA (Analysis of Variance). It is used to detect differences across several algorithms in multiple test attempts (datasets). For this test, we need to demonstrate that the Null-hypothesis-stating that there is no significant difference between different algorithms - is rejected (Demšar 2006).

The Friedman test is distributed according to Eq. 5 with $k-1$ degrees of freedom:

$$
\chi_{F}^{2}=\frac{12 N}{k(k+1)}\left[\sum_{j=1}^{k} R_{j}^{2}-\frac{k(k+1)^{2}}{4}\right],
$$

where $R_{j}$ is the rank of the $\mathrm{j}$-th of $k$ algorithms and $N$ is the number of datasets. Table 9 shows the average rank of each method included in the experiments in both the immediate and the delayed settings. 
Table 6 Accuracy (\%) of the methods compared in the immediate setting

\begin{tabular}{|c|c|c|c|c|c|c|c|}
\hline Dataset & Criteria & $\mathrm{ARF}$ & DWM & LevBag & OAUE & OSBoost & $E A C D_{E x p}$ \\
\hline \multirow[t]{4}{*}{ Hyper. } & Ave. & 88.17 & 89.64 & 91.03 & 91.42 & 85.85 & 90.59 \\
\hline & $\sigma$ & 1.90 & 0.83 & 1.60 & 1.46 & 3.01 & 1.95 \\
\hline & Min & 85.96 & 88.45 & 88.92 & 89.66 & 81.80 & 87.67 \\
\hline & $\operatorname{Max}$ & 91.31 & 90.94 & 93.54 & 93.63 & 89.87 & 93.76 \\
\hline \multirow[t]{4}{*}{ LED } & Ave. & 74.05 & 75.05 & 74.22 & 73.99 & 74.15 & 75.45 \\
\hline & $\sigma$ & 0.31 & 3.10 & 0.31 & 0.10 & 0.11 & 1.99 \\
\hline & Min & 73.58 & 73.86 & 73.93 & 73.89 & 74.05 & 71.04 \\
\hline & Max & 74.45 & 83.83 & 74.52 & 74.09 & 74.26 & 78.50 \\
\hline \multirow[t]{4}{*}{ RT } & Ave. & 78.35 & 59.35 & 90.78 & 88.88 & 93.40 & 91.42 \\
\hline & $\sigma$ & 8.12 & 8.87 & 2.26 & 3.26 & 1.45 & 2.82 \\
\hline & Min & 65.86 & 48.26 & 87.38 & 83.39 & 90.63 & 86.31 \\
\hline & Max & 88.03 & 73.86 & 93.72 & 92.56 & 95.24 & 94.56 \\
\hline \multirow[t]{4}{*}{ SEA } & Ave. & 88.67 & 87.72 & 87.59 & 88.69 & 85.56 & 90.08 \\
\hline & $\sigma$ & 0.58 & 0.57 & 1.67 & 0.58 & 0.35 & 2.94 \\
\hline & Min & 88.40 & 87.18 & 85.41 & 88.13 & 85.25 & 86.54 \\
\hline & $\operatorname{Max}$ & 89.55 & 88.26 & 89.28 & 89.25 & 85.86 & 93.74 \\
\hline \multirow[t]{4}{*}{ Airlines } & Ave. & 63.53 & 63.97 & 59.42 & 64.02 & 61.98 & 66.61 \\
\hline & $\sigma$ & 1.23 & 0 & 0.73 & 0 & 0 & 3.10 \\
\hline & Min & 62.08 & 63.97 & 58.45 & 64.02 & 61.98 & 60.34 \\
\hline & Max & 65.46 & 63.97 & 60.62 & 64.02 & 61.98 & 70.23 \\
\hline \multirow[t]{4}{*}{ Elec. } & Ave. & 92.17 & 75.73 & 92.09 & 91.60 & 88.02 & 92.14 \\
\hline & $\sigma$ & 0.94 & 0 & 1.48 & 0 & 0 & 1.76 \\
\hline & Min & 90.45 & 75.73 & 89.56 & 91.60 & 88.02 & 89.56 \\
\hline & Max & 93.19 & 75.73 & 93.70 & 91.60 & 88.02 & 94.72 \\
\hline \multirow[t]{4}{*}{ Forest } & Ave. & 93.57 & 83.75 & 92.73 & 90.70 & 84.45 & 91.73 \\
\hline & $\sigma$ & 1.58 & 0 & 2.10 & 0 & 0 & 3.10 \\
\hline & Min & 91.11 & 83.75 & 89.45 & 90.70 & 84.45 & 88.34 \\
\hline & Max & 95.09 & 83.75 & 95.40 & 90.70 & 84.45 & 95.12 \\
\hline \multirow[t]{4}{*}{ KDDcup } & Ave. & 99.81 & 99.04 & 99.82 & 99.80 & 99.74 & 99.78 \\
\hline & $\sigma$ & 0.06 & 0 & 0.01 & 0 & 0 & 0.10 \\
\hline & Min & 99.74 & 99.04 & 99.80 & 99.80 & 99.74 & 99.54 \\
\hline & Max & 99.91 & 99.04 & 99.83 & 99.80 & 99.74 & 99.85 \\
\hline \multirow[t]{4}{*}{ Poker } & Ave. & 84.19 & 74.37 & 88.52 & 80.74 & 84.31 & 86.21 \\
\hline & $\sigma$ & 4.55 & 0 & 3.34 & 0 & 0 & 2.37 \\
\hline & Min & 80.08 & 74.37 & 84.67 & 80.74 & 84.31 & 82.34 \\
\hline & $\operatorname{Max}$ & 90.06 & 74.37 & 93.56 & 80.74 & 84.31 & 89.34 \\
\hline
\end{tabular}

Best results are given in bold 
Table 7 Accuracy (\%) of the methods compared in the delayed setting

\begin{tabular}{|c|c|c|c|c|c|c|c|}
\hline Dataset & Criteria & $\mathrm{ARF}$ & DWM & LevBag & OAUE & OSBoost & $E A C D_{E x p}$ \\
\hline \multirow[t]{4}{*}{ Hyper. } & Ave. & 88.05 & 89.41 & 90.77 & 91.10 & 85.74 & 90.02 \\
\hline & $\sigma$ & 2.02 & 0.95 & 1.71 & 1.59 & 3.06 & 2.01 \\
\hline & Min & 85.56 & 88.25 & 88.60 & 89.21 & 81.70 & 86.64 \\
\hline & Max & 91.35 & 90.86 & 93.37 & 93.55 & 89.78 & 92.95 \\
\hline \multirow[t]{4}{*}{ LED } & Ave. & 74.00 & 74.14 & 74.21 & 74.06 & 74.13 & 75.26 \\
\hline & $\sigma$ & 0.40 & 0.16 & 0.15 & 0.14 & 0.04 & 1.33 \\
\hline & Min & 73.62 & 73.99 & 74.07 & 73.93 & 74.10 & 72.94 \\
\hline & Max & 74.49 & 74.30 & 74.36 & 74.19 & 74.17 & 77.04 \\
\hline \multirow[t]{4}{*}{ RT } & Ave. & 78.24 & 59.49 & 90.91 & 88.72 & 85.53 & 91.05 \\
\hline & $\sigma$ & 8.06 & 8.67 & 2.48 & 5.13 & 2.90 & 3.75 \\
\hline & Min & 65.81 & 49.16 & 86.94 & 82.17 & 81.17 & 84.64 \\
\hline & Max & 87.92 & 73.73 & 93.69 & 93.47 & 88.70 & 94.56 \\
\hline \multirow[t]{4}{*}{ SEA } & Ave. & 88.94 & 87.48 & 88.70 & 88.54 & 85.31 & 89.22 \\
\hline & $\sigma$ & 0.59 & 1.02 & 1.45 & 0.70 & 0.42 & 2.43 \\
\hline & Min & 88.28 & 86.01 & 86.89 & 87.81 & 84.92 & 86.04 \\
\hline & Max & 89.51 & 88.21 & 90.32 & 89.21 & 85.91 & 91.89 \\
\hline \multirow[t]{4}{*}{ Airlines } & Ave. & 61.42 & 60.57 & 58.49 & 62.73 & 61.80 & 63.35 \\
\hline & $\sigma$ & 1.12 & 0 & 0.89 & 0 & 0 & 3.78 \\
\hline & Min & 61.22 & 60.57 & 57.03 & 62.73 & 61.80 & 59.06 \\
\hline & Max & 63.32 & 60.57 & 59.65 & 62.73 & 61.80 & 68.34 \\
\hline \multirow[t]{4}{*}{ Elec. } & Ave. & 83.51 & 67.43 & 81.78 & $80.20 \%$ & 79.04 & 85.03 \\
\hline & $\sigma$ & 1.19 & 0 & 0.88 & 0 & 0 & 2.50 \\
\hline & Min & 81.78 & 67.43 & 80.54 & 80.20 & 79.04 & 80.45 \\
\hline & Max & 84.80 & 67.43 & 83.00 & 80.20 & 79.04 & 88.85 \\
\hline \multirow[t]{4}{*}{ Forest } & Ave. & 85.65 & 74.93 & 86.22 & 86.84 & 74.47 & 84.83 \\
\hline & $\sigma$ & 02.60 & 0 & 2.72 & 0 & 0 & 2.36 \\
\hline & Min & 83.67 & 74.93 & 84.30 & 86.84 & 74.47 & 81.45 \\
\hline & Max & 90.49 & 74.93 & 84.30 & 86.84 & 74.47 & 88.23 \\
\hline \multirow[t]{4}{*}{ KDDcup } & Ave. & 99.80 & 99.12 & 99.81 & 99.78 & 99.74 & 99.76 \\
\hline & $\sigma$ & 0.07 & 0 & 0.01 & 0 & 0 & 0.11 \\
\hline & Min & 99.72 & 99.12 & 99.79 & 99.78 & 99.74 & 99.48 \\
\hline & Max & 99.90 & 99.12 & 99.83 & 99.78 & 99.74 & 99.84 \\
\hline \multirow[t]{4}{*}{ Poker } & Ave. & 67.95 & 59.31 & 76.78 & 73.81 & 81.23 & 80.21 \\
\hline & $\sigma$ & 2.92 & 0 & 3.72 & 0 & 0 & 2.01 \\
\hline & Min & 64.94 & 59.31 & 70.51 & 73.81 & 81.23 & 76.35 \\
\hline & Max & 73.29 & 59.31 & 79.34 & 73.81 & 81.23 & 83.24 \\
\hline
\end{tabular}

Best results are given in bold 
Table 8 Average evaluation time (in seconds) of executing the methods compared in the immediate setting

\begin{tabular}{lllllll}
\hline Dataset & ARF & DWM & LevBag & OAUE & OSBoost & EACD Exp \\
\hline Hyperplane & 208 & 130 & 144 & 107 & $\mathbf{9 3}$ & 349 \\
LED & 188 & 851 & 246 & 227 & $\mathbf{1 7 4}$ & 423 \\
RT & 394 & 195 & 207 & $\mathbf{1 4 8}$ & 1141 & 607 \\
SEA & 751 & $\mathbf{9 8}$ & 409 & 139 & 162 & 880 \\
Airlines & 495 & $\mathbf{6 6}$ & 531 & 366 & 74 & 657 \\
Electricity & 7.73 & $\mathbf{1 . 4 8}$ & 5.12 & 3.05 & 2.06 & 10.45 \\
Forest & 153 & 148 & 206 & 180 & $\mathbf{1 1 4}$ & 935 \\
KDDcup99 & $\mathbf{5 6}$ & 581 & 130 & 204 & 138 & 536 \\
Poker & 167 & $\mathbf{4 6}$ & 81 & 66 & 64 & 346 \\
\hline
\end{tabular}

Best results are given in bold

Note that for each setting, $k=6$ and $N=9$, as there are six methods and nine different datasets. Providing the value of the Friedman test statistic is $\chi_{F}^{2}=12.49$ for the immediate setting and $\chi_{F}^{2}=17.38$ for the delayed setting with $5(k-1)$ degrees of freedom, and the critical value for the Friedman test given $k=6$ and $N=9$ is 10.78 at significance level $\alpha=0.05$, we can conclude that the accuracy values of the studied methods are significantly different in both settings as their $\chi_{F}^{2}$ values (12.49 and 17.38) are greater than the critical value (10.78).

Now that the Null-hypothesis is rejected, we can proceed with a post-hoc test. The Nemenyi test (Nemenyi 1962) can be used when several classifiers are compared to each other (Demšar 2006). The performance of two classifiers is significantly different if their corresponding average ranks differ by at least the critical difference (CD).

The critical value in our experiments with $k=6$ and $\alpha=0.10$ is $q_{0.10}=2.28$. As a result, the accuracy of the proposed $E A C D_{E x p}$ method is significantly different from that of DWM and OSBoost, whereas it is not significantly different from LevBag, ARF and OAUE. Figure 4 graphically represents the comparison of the methods in both settings based on the Nemenyi test.

\section{Discussion}

As can be observed from Tables 3, 4 and 5, the average accuracy values of the explicit variations $\left(E A C D_{E x p}\right.$ and $\left.E A C D_{E x p 2}\right)$ are slightly better than that of the implicit variations $\left(E A C D_{I m p}\right.$ and $\left.E A C D_{\operatorname{Imp} 2}\right)$. Furthermore, the accuracy values of the variations that use the GA optimisation technique are significantly better than that the base variations for all datasets. By looking at the results of $E A C D_{\text {base }}, E A C D_{\text {base }} 4$ and $E A C D_{E x p}$, it can be concluded that using a concept drift detection mechanism alone cannot improve the results significantly, whereas using the concept drift detector along with a stochastic optimiser (GA) improves the accuracy significantly.

Among the variations that use only the base layer of the proposed algorithm, those that use a higher number of types and a higher number of features in each type ( $E A C D_{\text {base } 4}$ and $E A C D_{\text {base }}$ ) are performing better compared to the other varia- 

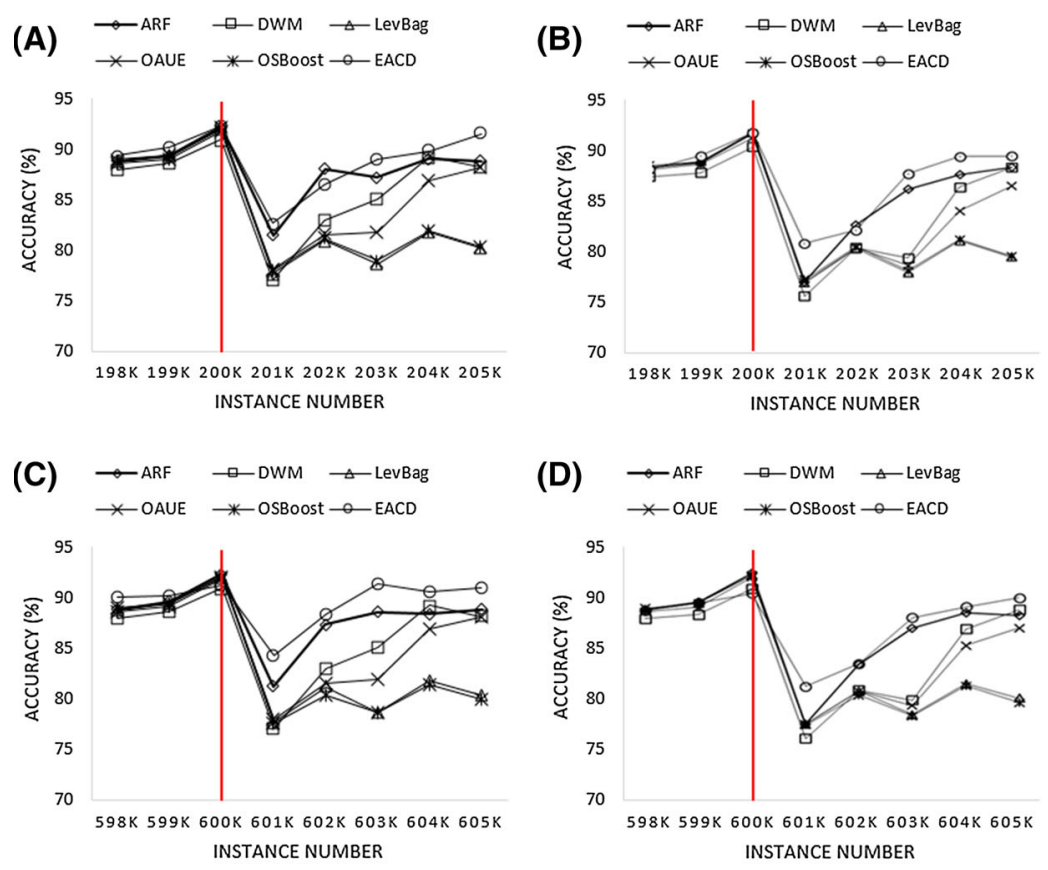

(E) $\rightarrow$ ARF $\rightarrow$ DWM $\triangle$ LevBag
$\rightarrow$ OAUE $\rightarrow$ OSBoost - - EACD
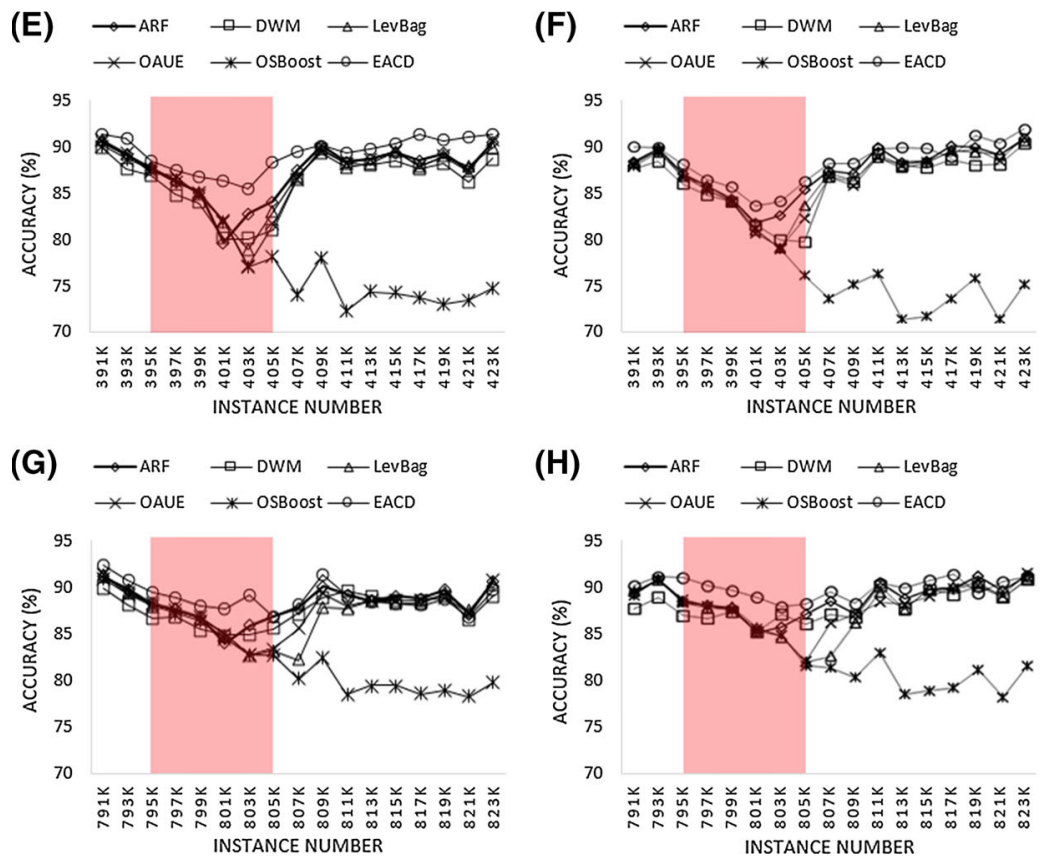

Fig. 3 Behaviour of the methods compared upon different concept drifts added to the SEA dataset in the immediate setting (left column; a, c, e, g) and the delayed setting (right column; b, d, f, h). The red boxes indicate the location and the length of the added concept drifts 
Table 9 Average rank of the methods compared

\begin{tabular}{lllllll}
\hline Setting & ARF & DWM & LevBag & OAUE & OSBoost & EACDExp \\
\hline Immediate & 3.33 & 4.78 & 2.89 & 3.44 & 4.44 & $\mathbf{2 . 1 1}$ \\
Delayed & 3.78 & 5.11 & 2.67 & 3 & 4.44 & $\mathbf{2}$ \\
\hline
\end{tabular}

Best results are given in bold
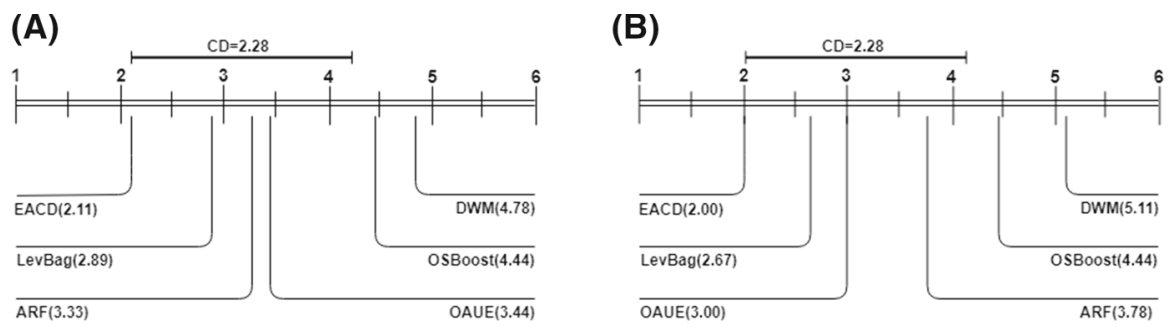

Fig. 4 Nemenyi test with $90 \%$ confidence level for a immediate and $\mathbf{b}$ delayed setting

tions in the majority of the experiments. This is because the former variations create more classifiers on each time-step, with each classifier covering more features itself. This also justifies why they are more time consuming compared to the other base-layer variations. Furthermore, when using a concept drift detection mechanism along with the base layer in $E A C D_{\text {base }}$ variation, it fails to improve the accuracy significantly compared to the variation with the same parameters but without using a concept drift detector in $E A C D_{\text {base }}$ (improving only by $0.25 \%$ in the immediate and by $0.33 \%$ in the delayed setting). The explanation for this might be that while concept drift detectors can be very helpful for achieving a fast reaction to evolving data, they can also be destructive upon false alarms, especially when trained classifiers are removed immediately upon concept drifts.

While the average accuracy of the explicit variations is significantly better than that of the base variations, their execution time is significantly longer than that of the base variations in all experiments. This is because the base variations use only the first layer of the proposed architecture and not the genetic layer, unlike the implicit and the explicit variations that use both layers. Furthermore, since the combination of the features in random subspaces (types) in the base variations is not optimised during the run, and only the number of classifiers in each subspace is changed, the overall accuracy depends greatly on the initial selection of the features. In the implicit and the explicit variations however, the combination of the features in each subspace is reconstructed by GA when needed.

The difference between the implicit and the explicit variations of the proposed method is the time it takes them to decide when to let GA start optimising a set of subspaces using the buffer of recently stored instances. Since the average accuracy of $E A C D_{E x p}$ is about $1.13 \%$ higher than that of $E A C D_{\text {Imp }}$ in the immediate setting and $1.02 \%$ higher in the delayed setting, we can conclude that one of the most challenging parts of the proposed architecture is to decide when GA needs to reconstruct the combination of classification types in the genetic layer. 
When looking at the values of the standard deviation for the real-world datasets used in the experiments (Tables 6, 7), it can be noticed that DWM, OAUE and OSBoost have the same standard deviation of zero for all real-world datasets, whereas RD3+GA, LevBag and ARF have different standard deviation values. This is because the latter algorithms use randomisation in their procedures, whereas the former do not. Since the experiments over the real-world datasets are repeated 10 times over the same data, the results obtained from all deterministic algorithms in all iterations are the same.

It can be further noticed from Tables 6 and 7 that for the artificial datasets, the standard deviation values for OSBoost, OAUE and DWM vary greatly throughout the experiments, reaching the value of about $8 \%$ for the RT dataset. At the same time, the standard deviation values for LevBag, ARF and $E A C D_{E x p}$ do not vary a lot, hardly reaching the value of $3.78 \%$. This might be because the first three methods (OSBoost, OAUE and DWM) are implicit and do not use any concept drift detection mechanisms, whereas the other methods (LevBag, ARF and $E A C D_{\text {Exp }}$ ) are explicit and use concept drift detection mechanisms. As explicit methods have an immediate reaction to concept drifts, their accuracy does not drop for a long time throughout the experiments.

Form Table 8, it can be noticed that DWM has the lowest evaluation time over four datasets, OSBoost - over three datasets, whereas ARF and OAUE-over one dataset. The main drawback of the $E A C D_{E x p}$ variation of the proposed algorithm is its evaluation time, which is the longest for the majority of the datasets (six out of nine). The main reason for this is that this variation uses two different evolutionary algorithms (RD and GA) along with a concept drift detection method (EDDM). However, the other variations of the proposed algorithm offer slightly shorter evaluation times in $E A C D_{\text {Imp }}$ and $E A C D_{I m p 2}$, and significantly shorter times in $E A C D_{\text {base }}, E A C D_{\text {base } 2}$ and $E A C D_{\text {base3 }}$. This is because the implicit variations of the EACD algorithm use both evolutionary algorithms but no concept drift detection method, while the base variations use only one evolutionary algorithm (RD) with no drift detection method.

In Fig. 3a, where an abrupt concept drift has occurred, the EAC $D_{\text {Exp }}$ and ARF methods coped with the drift better than the other methods with almost similar reactions. The same can be observed in the delayed setting for the same drift (Fig. 3b); however, the accuracy drop upon the drift is more drastic in ARF compared to $E A C D_{E x p}$. The reason for this might be their explicit strategy allowing to detect concept drifts as soon as they occur and use their recovery mechanism. In addition, detecting abrupt concept drifts should be easier for the concept drift detectors as the data distribution changes suddenly in such drifts. Furthermore, using different random types in the base layer of $E A C D_{E x p}$ can result in a more robust performance, especially over drifting data, when the data distribution is not known in advance. DWM, OAUE and LevBag cope with concept drifts more slowly compared to ARF and $E A C D_{E x p}$, while OSBoost seems to fail to adapt to the introduced abrupt concept drift in a good time.

In Fig. 3c, d, where a recurrent concept drift (with a width of one) occurred in the instance number $600 \mathrm{~K}$, the accuracy of all methods dropped, with $E A C D_{E x p}$ taking less time to adapt to the new data distribution and gain its average accuracy back again in both the immediate and the delayed settings. This might be because the proposed method uses two different mechanisms to cope with new environments: 
one (RD) weights the classification types based on their performance, while the other (GA) optimises the combination of the attributes of these types.

In Fig. 3e, f, where a gradual concept drift (with a width of 10,000 and centred in the instance number $400 \mathrm{~K}$ ) occurred, it is clear that $E A C D_{E x p}$ copes with this concept drift in a more robust manner compared to the other methods in both settings. In the situations when a concept drift happens gradually, the time of detecting the drift plays an important role in how the drift is addressed, since the majority of the explicit methods start their adaptation procedure at that time. Hence, failing to detect the drift on time can cause the methods to suffer from the late adaptation. In the proposed method however, adaptation to the drifts can be divided into two stages: (1) before the drift is detected, when the algorithm tries to seamlessly adapt to the drift using RD; and (2) after the drift is detected, when GA starts to optimise the combination of the attributes in the genetic layer. This justifies the better performance of the proposed method, especially upon gradual concept drifts.

In Fig. 3g, where a recurrent concept drift (with a width of 10,000) occurred in the immediate setting, the accuracy of all methods dropped within the same rate. However, $E A C D_{E x p}$ took less time to adapt compared to the other methods. In Fig. 3h, where the the same drift is shown in the delayed setting, the behaviour of all methods except OSBoost is relatively similar; however, the accuracy of $E A C D_{E x p}$ degrades less than that of the other methods during the drifting period (shown by the red box). In both settings, OSBoost fails to continue improving its performance for at least 14,000 instances from the instance number $805 \mathrm{~K}$. This behaviour of OSBoost is similar to its results upon abrupt and gradual concept drifts, which shows that the method lacks a sound adaptation mechanism over different types of concept drifts.

Overall, the main advantage of the proposed $E A C D_{\text {Exp }}$ method is its accuracy; it has the best average rank compared to the other state-of-the-art methods used in the experiments (as shown in Table 9). It also proved to have the fastest reaction over evolving data on most occasions, especially upon abrupt, gradual and recurrent concept drifts, as shown in Fig. 3.

While the proposed method is specifically designed to cope with non-stationary environments, it is possible to use it in stationary environments. However, the main limitation in this case would be the unnecessary overhead that the algorithm puts on the ensemble since the algorithm always builds classifiers over different time-stamps of the target data stream, while there is no need to do that, when a data stream does not evolve.

\section{Conclusion and future work}

In this paper, we proposed a novel method to seamlessly adapt to concept drifts in nonstationary data stream classification. The Evolutionary Adaptation to Concept Drifts (EACD) method has two layers with a set of classifiers in each layer. The first layer (base layer) is constructed by creating randomly drawn set of subspaces (classification types) from the pool of features of the target data stream. Each type is the basis for building decision trees (classifiers) in a layer. To seamlessly adapt to concept drifts in our approach, the replicator dynamics algorithm is used to increase or reduce the 
number of trees in each type according to their recent performance in the data stream. The second layer (genetic layer) uses randomly drawn subspaces from the first layer as the first population for Genetic Algorithm employed to optimise the classification types with the most recent instances. Creating new classifiers and training the current classifiers in this layer is the same as in the base layer. For the genetic layer, two different mechanisms are proposed to determine when to restart Genetic Algorithm. The first mechanism is based on comparing the performance of the two layers (implicit EACD), whereas the second one uses a concept drift detection method to check when a new concept drift occurs (explicit EACD).

To test the proposed method and its variations, a set of experiments with five realworld and four artificial datasets was conducted. First, the performance of different variations of the proposed method was compared; then, the best performing variation was compared to the state-of-the-art methods proposed in the literature. All experiments were conducted in two different settings: the immediate prequential and the delayed prequential. The results showed that our method achieves the highest average accuracy and the best average rank among all methods in both settings. However, the overall evaluation time of the proposed method is the longest in six out of nine datasets, which makes the evaluation time to be the main drawback of EACD.

Using the Friedman statistical test, it was shown that the accuracy values of the studied methods are significantly different, and according to the Nemenyi test (which is a post-hoc test of the Friedman test), the accuracy of the proposed $E A C D_{\text {exp }}$ method is significantly different from that of DWM and OSBoost, while it is not significantly different from that of ARF, LevBag and OAUE.

The presented work opens the door to new developments that need to be theoretically analysed and practically tested in the future. The following ideas are proposed, to mention some.

- Detecting the classification types that have not been useful for a long time in different environments to remove them and eventually make a room for new, better performing types to be added.

- Using dynamic instead of static weights for the base and the genetic layers of the method to have a potentially more robust weighting mechanism.

- Using a different removal mechanism when the maximum number of trees for a classification type is reached and a classifier (decision tree) should be removed; e.g. removing the oldest classifier inside the type instead of removing the worst performing one, as it is proposed in this paper.

- Adding the good performing classification types that are produced in the genetic layer to the base layer to keep them in the ensemble since the later layer can be cleared after some time. This can help optimising the algorithm, especially regarding the time criterion.

- Developing a pattern recognition system to track the usability of each type in different environments. This can lead to knowing the types better and using such information when data evolve, especially when a recurring concept drift occurs.

- Introducing a new concept drift detection system by analysing the behaviour of the classification types. 
Open Access This article is distributed under the terms of the Creative Commons Attribution 4.0 International License (http://creativecommons.org/licenses/by/4.0/), which permits unrestricted use, distribution, and reproduction in any medium, provided you give appropriate credit to the original author(s) and the source, provide a link to the Creative Commons license, and indicate if changes were made.

\section{References}

Baena-Garcia M, del Campo-Avila J, Fidalgo R, Bifet A, Gavalda R, Morales-Bueno R (2006) Early drift detection method. In: Proceedings of the 4th ECMLPKDD international workshop on knowledge discovery from data streams, pp 77-86

Bifet A, Gavalda R (2007) Learning from time-changing data with adaptive windowing. In: Proceedings of the 2007 SIAM international conference on data mining, pp 443-448. SIAM

Bifet A, Holmes G, Pfahringer B, Kirkby R, Gavaldà R (2009) New ensemble methods for evolving data streams. In: Proceedings of the 15th ACM SIGKDD international conference on Knowledge discovery and data mining, pp 139-148. ACM

Bifet A, Holmes G, Kirkby R, Pfahringer B (2010a) Moa: massive online analysis. J Mach Learn Res 11(May):1601-1604

Bifet A, Holmes G, Pfahringer B (2010b) Leveraging bagging for evolving data streams. In: Machine learning and knowledge discovery in databases, pp 135-150

Blackard JA, Dean DJ (1999) Comparative accuracies of artificial neural networks and discriminant analysis in predicting forest cover types from cartographic variables. Comput Electron Agric 24(3):131-151

Bomze IM (1983) Lotka-Volterra equation and replicator dynamics: a two-dimensional classification. Biol Cybern 48(3):201-211

Breiman L (2001) Random forests. Mach Learn 45(1):5-32

Breiman L, Friedman J, Stone CJ, Olshen RA (1984) Classification and regression trees. CRC Press, Boca Raton

Brzezinski D, Stefanowski J (2014a) Combining block-based and online methods in learning ensembles from concept drifting data streams. Inf Sci 265:50-67

Brzezinski D, Stefanowski J (2014b) Reacting to different types of concept drift: the accuracy updated ensemble algorithm. IEEE Trans Neural Netw Learn Syst 25(1):81-94

Chen S-T, Lin H-T, Lu C-J (2012) An online boosting algorithm with theoretical justifications. arXiv preprint arXiv: 1206.6422

Chu F, Zaniolo C (2004) Fast and light boosting for adaptive mining of data streams. In: Pacific-Asia conference on knowledge discovery and data mining, pp 282-292. Springer

Cup KDD 1999 Data (1999) http://kdd.ics.uci.edu/databases/kddcup99/kddcup99.html. Accessed 23 Mar 2018

Domingos P, Hulten G (2000) Mining high-speed data streams. In: Proceedings of the sixth ACM SIGKDD international conference on Knowledge discovery and data mining, pp 71-80. ACM

Elwell R, Polikar R (2011) Incremental learning of concept drift in nonstationary environments. IEEE Trans Neural Netw 22(10):1517-1531

Elyan E, Gaber MM (2017) A genetic algorithm approach to optimising random forests applied to class engineered data. Inf Sci 384:220-234

Fawgreh K, Gaber MM, Elyan E (2015) A replicator dynamics approach to collective feature engineering in random forests. In: Research and development in intelligent systems XXXII, pp 25-41. Springer

Folino G, Pizzuti C, Spezzano G (2006) GP ensembles for large-scale data classification. IEEE Trans Evol Comput 10(5):604-616

Folino G, Pizzuti C, Spezzano G (2007) An adaptive distributed ensemble approach to mine concept-drifting data streams. In: 19th IEEE international conference on tools with artificial intelligence, ICTAI 2007, vol 2, pp 183-188. IEEE

Friedman M (1940) A comparison of alternative tests of significance for the problem of m rankings. Annals Math Stat 11(1):86-92

Gama J, Žliobaitė I, Bifet A, Pechenizkiy M, Bouchachia A (2014) A survey on concept drift adaptation. ACM Comput Surv (CSUR) 46(4):44

Gen M, Cheng R (2000) Genetic algorithms and engineering optimization, vol 7. Wiley, London 
Gomes HM, Enembreck F (2013) SAE: social adaptive ensemble classifier for data streams. In: 2013 IEEE symposium on computational intelligence and data mining (CIDM), pp 199-206. IEEE

Gomes HM, Enembreck F (2014) SAE2: advances on the social adaptive ensemble classifier for data streams. In: Proceedings of the 29th annual ACM symposium on applied computing, pp 798-804. $\mathrm{ACM}$

Gomes HM, Barddal JP, Enembreck F, Bifet A (2017a) A survey on ensemble learning for data stream classification. ACM Comput Surv (CSUR) 50(2):23

Gomes HM, Bifet A, Read J, Barddal JP, Enembreck F, Pfharinger B, Holmes G, Abdessalem T (2017b) Adaptive random forests for evolving data stream classification. Mach Learn 106:1-27

Gonçalves PM Jr, De Barros RSM (2013) RCD: a recurring concept drift framework. Pattern Recogn Lett 34(9):1018-1025

Harries M (1999) Splice-2 comparative evaluation: electricity pricing. Technical report, The University of South Wales

Hofbauer J, Sigmund K (2003) Evolutionary game dynamics. Bull Am Math Soc 40(4):479-519

Hulten G, Spencer L, Domingos P (2001) Mining time-changing data streams. In: Proceedings of the seventh ACM SIGKDD international conference on Knowledge discovery and data mining, pp 97-106. ACM

Jaber G (2013) An approach for online learning in the presence of concept change. PhD thesis, Citeseer

Janez D (2006) Statistical comparisons of classifiers over multiple data sets. J Mach Learn Res 7(Jan):1-30

Kolter JZ, Maloof MA (2007) Dynamic weighted majority: an ensemble method for drifting concepts. J Mach Learn Res 8(Dec):2755-2790

Krawczyk B, Minku LL, Gama J, Stefanowski J, Woźniak M (2017) Ensemble learning for data stream analysis: a survey. Inf Fusion 37:132-156

Mantri A, Kendra SNS, Kumar G, Kumar S (2011) Proceedings of the high performance architecture and grid computing: international conference, HPAGC 2011, Chandigarh, India, 19-20 July 2011 , vol 169. Springer

Nemenyi P (1962) Distribution-free multiple comparisons. In: Biometrics, vol 18, p 263. International Biometric Society

Oza NC (2005) Online bagging and boosting. IEEE Int Conf Syst Man Cybernet 3:2340-2345

Ramamurthy S, Bhatnagar R (2007) Tracking recurrent concept drift in streaming data using ensemble classifiers. In: Sixth international conference on machine learning and applications, ICMLA 2007, pp 404-409. IEEE

Spall JC (2005) Introduction to stochastic search and optimization: estimation, simulation, and control, vol 65. Wiley, London

Street WN, Kim YS (2001) A streaming ensemble algorithm (SEA) for large-scale classification. In: Proceedings of the seventh ACM SIGKDD international conference on Knowledge discovery and data mining, pp 377-382. ACM

Vivekanandan P, Nedunchezhian R (2011) Mining data streams with concept drifts using genetic algorithm. Artif Intell Rev 36(3):163-178

Publisher's Note Springer Nature remains neutral with regard to jurisdictional claims in published maps and institutional affiliations. 